\title{
Understanding the evolution of multiple drug resistance in structured populations
}

\author{
David V. McLeod ${ }^{* 1}$ and Sylvain Gandon ${ }^{\dagger 1}$ \\ ${ }^{1}$ Centre D’Ecologie Fonctionnelle \& Evolutive, CNRS, Montpellier, France
}

\begin{abstract}
The evolution of multidrug resistance (MDR) is a pressing public health concern. Yet many aspects, such as the role played by population structure, remain poorly understood. Here we argue that studying MDR evolution by focusing upon the dynamical equations for linkage disequilibrium (LD) can greatly simplify the calculations, generate more insight, and provide a unified framework for understanding the role of population structure. We demonstrate how a general epidemiological model of MDR evolution can be recast in terms of the LD equations. These equations reveal how the different forces generating and propagating LD operate in a dynamical setting at both the population and metapopulation levels. We then apply these insights to show how the LD perspective: (i) explains equilibrium patterns of MDR, (ii) provides a simple interpretative framework for transient evolutionary dynamics, and (iii) can be used to assess the consequences of different drug prescription strategies for MDR evolution.
\end{abstract}

Keywords: antibiotic resistance, multidrug resistance, linkage disequilibrium, evolutionary epidemiology

*david.mcleod@cefe.cnrs.fr

†sylvain.gandon@cefe.cnrs.fr 


\section{Introduction}

Antibiotic resistance is one of the biggest current public health problems, with antibiotic resistant infections responsible for tens of thousands of deaths annually (O'Neill 2015). Of particular concern is the evolution of multidrug resistant (MDR) pathogens, that is, pathogens resistant to multiple classes of antibiotics. Despite its importance, understanding the evolution of MDR remains an ongoing challenge, as it is typically not captured by our understanding of the evolution of single drug resistance (for which there is a large body of theory; e.g., Austin and Anderson 1999: Bergstrom et al.|2004; Blanquart 2019; Bonhoeffer et al.|1997; Lipsitch et al.|2000). For instance, suppose we have two drugs, $A$ and $B$, and that a fraction $f_{A B}$ of infections caused by the pathogen of interest are resistant to both drugs. To understand MDR evolution, we need to understand what determines the frequency $f_{A B}$. If $f_{A}$ and $f_{B}$ are the frequency of infections resistant to drug $A$ and $B$, and $D$ denotes any non-random association between resistance to drugs $A$ and $B$, then

$$
f_{A B}=f_{A} f_{B}+D
$$

If $D=0$, then the evolution of resistance to each drug is independent, and so multiple drugs do not qualitatively alter the evolutionary dynamics of single drug resistance. However, whenever $D \neq 0$, understanding the fitness costs and benefits of resistance to each drug in isolation is insufficient to understand the evolution of MDR, because doing so will not tell us what factors govern the propagation of $D$, which in turn will affect $f_{A}$ and $f_{B}$. Thus the challenge of understanding MDR evolution can be recast as understanding the dynamics of $D$. As it turns out, the quantity $D$ is referred to as linkage disequilibrium (LD), and it has been extensively studied in population genetics (e.g., Barton 1995; Felsenstein 1965: Lewontin 1964a: Ohta 1982a; Rice 2004: Slatkin 2008), particularly as it relates to population structure (Lenormand and Otto 2000; Li and Nei 1974; Martin et al. 2006; Nei and Li 1973; Ohta 1982b; Slatkin 1975). However, there has been little attempt to apply these insights to MDR evolution; often the dynamics of doubly-resistant infections are neglected to simplify the analysis of single drug resistance (e.g., Beardmore et al.2017, Bergstrom et al.|2004; Bonhoeffer et al.|1997).

Here we consider a simple epidemiological model of a primarily asymptomatically carried pathogen (e.g., Staphylococcus spp. or Enterococcus spp.) in a structured host population. We show how this model relates to general dynamical equations for LD (Day and Gandon 2012), in turn revealing the role of population structure in MDR evolution. We then use these equations to show how analyzing problems from the LD perspective: (i) reveals the evolutionary logic underlying patterns of MDR at equilibrium, which we use to reexamine a recent paper on MDR evolution (Lehtinen et al. 2019); (ii) provides a framework for understanding transient evolutionary dynamics; and (iii) provides insight on the consequences different drug prescription strategies have on MDR, which we apply to a hospital-community setting.

\section{Model}

In what follows we will introduce and analyze a model of MDR evolution. We will highlight the most important aspects here while providing more extensive details in the Sup. Mat. 1 .

Consider an asymptomatically carried pathogen in a metapopulation consisting of $N$ host populations. Focus upon population $x$. Let $S^{x}$ and $I_{i j}^{x}$ denote the density of susceptible hosts 
and $i j$-infections, respectively, at time $t$, where $i$ indicates if the infection is resistant $(i=A)$ or not $(i=a)$ to drug $A$ and $j$ indicates if the infection is resistant $(j=B)$ or not $(j=b)$ to $\operatorname{drug} B$. Susceptible hosts contract $i j$-infections at a per-capita rate $\beta_{i j}^{x} I_{i j}^{x}$, where $\beta_{i j}^{x}$ is a rate constant, while $i j$-infections are naturally cleared at a per-capita rate $\alpha_{i j}^{x}$. Hosts are treated with drugs $A, B$, or both in combination, at per-capita rates $\tau_{A}^{x}$, $\tau_{B}^{x}$, and $\tau_{A B}^{x}$, respectively. Treatment is instantaneous and resistance is complete, that is, if the host that receives treatment is infected by a strain sensitive to the drug, the infection is cleared instantaneously, whereas if the host which receives treatment is infected by a strain resistant to the drug, treatment has no effect. Hosts move from population $x$ to $y$ at a per-capita rate $m^{x \rightarrow y}$. Transmission between infected hosts leads to superinfection with probability $\sigma$, in which either strain is equally likely to be the victor. Finally, individual infections acquire allele $i$ through either mutation or recombination (during superinfection) at per-capita rates $\mu_{i}^{x}$ and $\rho_{i}^{x}$, respectively (note that $\rho_{i}^{x}$ depends upon infection densities, see Sup. Mat. equation (13)).

From these epidemiological assumptions, the change in $i j$-infections in population $x$ can be written as the sum of four processes

$$
\frac{\mathrm{d} I_{i j}^{x}}{\mathrm{dt}}=\overbrace{\left(r^{x}+\mathbf{1}_{A} s_{A}^{x}+\mathbf{1}_{B} s_{B}^{x}+\mathbf{1}_{A} \mathbf{1}_{B} s_{E}^{x}\right.}^{\text {per-capita growth }}) I_{i j}^{x}+\overbrace{\phi \mu_{i j}^{x}}^{\text {mutation }}+\overbrace{\phi \rho_{i j}^{x}}^{\text {recombination }}+\overbrace{\sum_{\substack{y=1 \\ y \neq x}}^{N}\left(m^{y \rightarrow x} I_{i j}^{y}-m^{x \rightarrow y} I_{i j}^{x}\right)}^{\text {migration }},
$$

where $\mathbf{1}_{d}$ is equal to 1 if the $i j$-infection is resistant to drug $d$ and 0 otherwise (e.g., if $i j=A B$, then the per-capita growth is $\left.r^{x}+s_{A}^{x}+s_{B}^{x}+s_{E}^{x}\right)$ and $\phi \mu_{i j}^{x}$ and $\phi \rho_{i j}^{x}$ denote the net change in $i j$ infections due to mutation and recombination (Fig. 1). To faciliate comparison with previous results, we have broken the per-capita growth term into four components: the 'baseline' percapita growth rate, $r^{x}$, the (additive) selection coefficients for resistance to drugs $A$ and $B, s_{A}^{x}$ and $s_{B}^{x}$, and any epistatic interactions, $s_{E}^{x}$. These latter terms have the standard interpretation. If $s_{A}^{x}>0$ (resp. $s_{B}^{x}>0$ ), then resistance to drug $A$ (resp. $B$ ) is selected for. If $s_{E}^{x}>0$, there is positive epistasis, and the per-capita growth rate of doubly-resistant infections is greater than would be expected by consideration of the per-capita growth rate of singly-resistant infections. Thus although equation (2) is derived from a specific model, the partitioning is very general and applies to many epidemiological scenarios. We stress that any of the terms $s_{d}^{x}, s_{E}^{x}, \phi \mu_{i j}^{x}$, and $\phi \rho_{i j}^{x}$ may themselves depend upon population densities (see Fig. 1 for a concrete example).

While system (2) contains all of the information necessary to analyze MDR evolution, as currently written it is particularly opaque for providing insight. Therefore we would like to transform it to a form which brings to the forefront the different factors that promote or impede MDR evolution; the way to do this is by focusing upon the dynamical equations for linkage disequilibrium (LD) (Day and Gandon|2012: Slatkin|2008). However, the inclusion of multiple populations means that doing so is not as simple as equation (1) would suggest since there are different scales at which LD and MDR can be measured. As the scale which is of most interest will depend upon the specifics of the problem, in what follows we will consider MDR evolution at both the population- and metapopulation-level. 


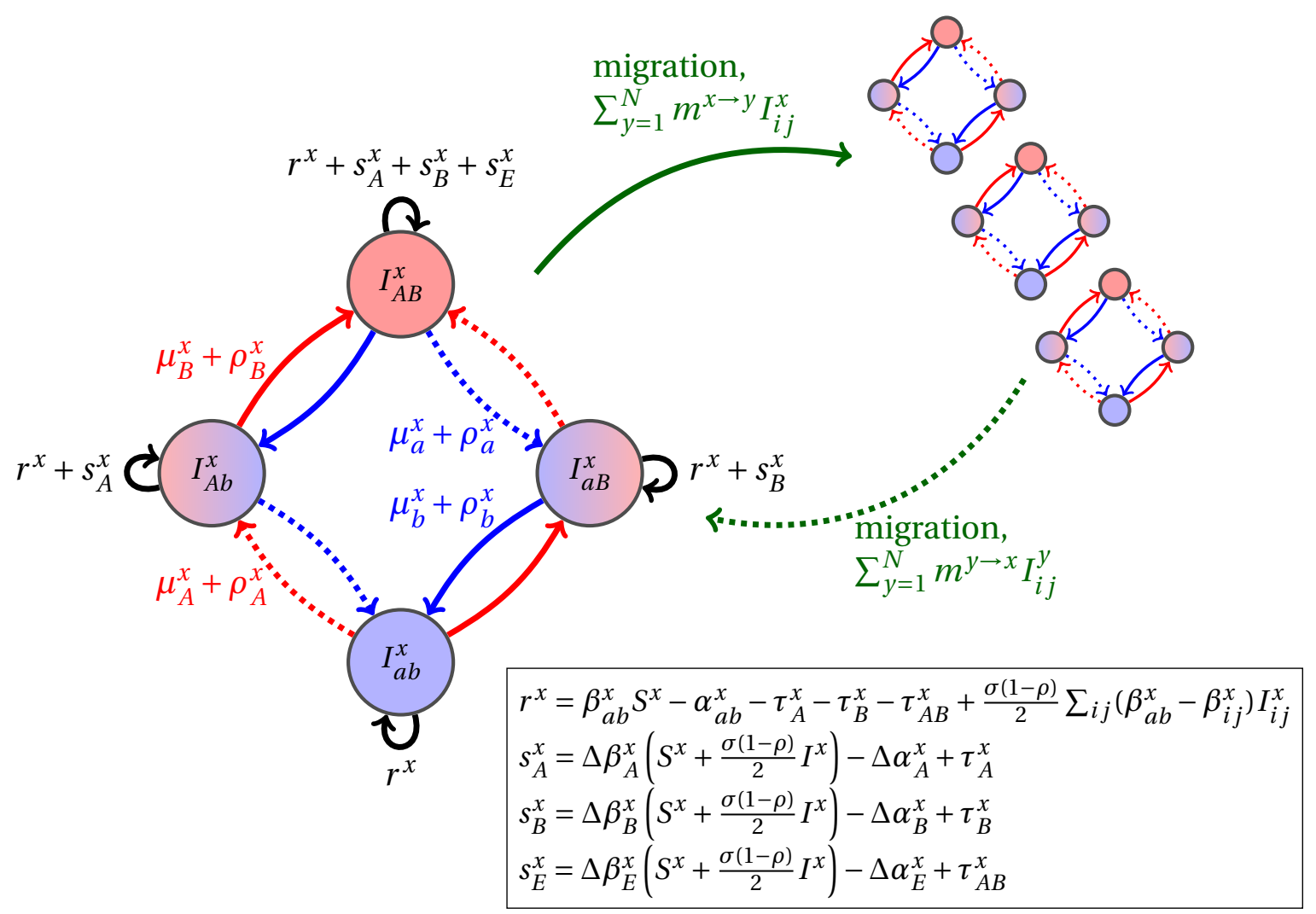

Figure 1: Schematic of the dynamics of system (2). The metapopulation consists of $N$ connected populations. Each population has four possible types of infections, linked by one-step mutation or recombination (blue and red arrows), whose per-capita rates are independent of genetic background. The 'baseline' per-capita growth rate of sensitive infections is $r^{x}$, the additive selection coefficients for $\operatorname{drug} A$ and $B$ resistance are $s_{A}^{x}$ and $s_{B}^{x}$, respectively, while $s_{E}^{x}$ denotes any epistatic interactions. In the inset, we compute these quantities for the specific model introduced in the main text, using the notation that $\Delta z_{d}^{x}$ and $\Delta z_{E}^{x}$ are the contribution of trait $z$ to the additive selection coefficient (for resistance to drug $d$ ) and epistasis, respectively, in population $x$ (e.g., $\Delta \beta_{A}^{x}=\beta_{A b}^{x}-\beta_{a b}^{x}$ and $\Delta \beta_{E}^{x}=\beta_{A B}^{x}-\beta_{a b}^{x}-\Delta \beta_{A}^{x}-\Delta \beta_{B}^{x}$ ).

\section{Population-level multidrug resistance}

To understand MDR evolution in a given population, say $x$, we need to understand the dynamics of the frequency of infections resistant to drug $A$ and $B, f_{A}^{x}$ and $f_{B}^{x}$, and the dynamics of population LD, $D^{x}$. First, consider the dynamics of $f_{A}^{x}$ (mutatis mutandis $f_{B}^{x}$ ). Using equation 
81

(2), it is straightforward to compute

$$
\begin{aligned}
\frac{\mathrm{d} f_{A}^{x}}{\mathrm{~d} t}=\overbrace{s_{A}^{x} f_{A}^{x}\left(1-f_{A}^{x}\right)}^{\text {direct selection }}+\overbrace{s_{B}^{x} D^{x}}^{\text {indirect }}+\overbrace{s_{E}^{x} f_{A}^{x}\left(1-f_{A}^{x}\right) \frac{f_{A B}^{x}}{f_{A}^{x}}}^{\text {epistasis }} & \\
& +\underbrace{\left(\mu_{A}^{x}+\rho_{A}^{x}\right)\left(1-f_{A}^{x}\right)-\left(\mu_{a}^{x}+\rho_{a}^{x}\right) f_{A}^{x}}_{\text {mutation and recombination }}-\underbrace{\sum_{y=1}^{N} m^{y \rightarrow x} \frac{I^{y}}{I^{x}}\left(f_{A}^{x}-f_{A}^{y}\right)}_{\text {migration }} .
\end{aligned}
$$

where $I^{x}$ is the total density of infections in population $x$ and $f_{A B}^{x}=D^{x}+f_{A}^{x} f_{B}^{x}$ is the frequency of doubly-resistant infections. A related formulation to equation (3) can be found in Day and Gandon (2012) (see also Rice 2004).

Equation (3) is partitioned into recognizable quantities. First, if resistance to drug $A$ is selectively advantageous, $s_{A}^{x}>0$, then $\operatorname{drug} A$ resistance will increase due to direct selection whose strength is dictated by the genetic variance at the locus, $f_{A}^{x}\left(1-f_{A}^{x}\right)$ (Fisher 1930). Second, if doubly-resistant infections are over-represented in the population, $D^{x}>0$, and resistance to $\operatorname{drug} B$ is selected for, $s_{B}^{x}>0$, then $\operatorname{drug} A$ resistance will increase due to indirect selection upon resistance to drug $B$. Third, if epistasis is positive, $s_{E}^{x}>0$, and there is genetic variance at the locus, drug $A$ resistance will increase due to the disproportionate growth of doubly-resistant infections. Fourth, mutation and recombination will increase drug $A$ resistance when there is a mutation or recombination bias towards gain of $\operatorname{drug} A$ resistance, $\mu_{A}^{x}>\mu_{a}^{x}$ or $\rho_{A}^{x}>\rho_{a}^{x}$, and the frequency of infections sensitive to drug $A$ exceeds the frequency of infections resistant to drug $A, 1-f_{A}^{x}>f_{A}^{x}$. Finally, migration acts to reduce differences between populations.

It follows that drug $B$ treatment alters the predicted dynamics of resistance to drug $A$ via two main effects: (i) the influence of epistasis and (ii) indirect selection on resistance to drug $B$ mediated through the presence of $\operatorname{LD}\left(D^{x} \neq 0\right)$. Thus consider the dynamics of $D^{x}$,

$$
\begin{aligned}
\frac{\mathrm{d} D^{x}}{\mathrm{dt}}=\overbrace{\left(s_{A}^{x}-s^{x}+s_{B}^{x}-s^{x}\right) D^{x}}^{\text {selection }}-\overbrace{\left(\mu^{x}+\rho^{x}\right) D^{x}}^{\text {mutation and recombination }} & \\
+\underbrace{s_{E}^{x} f_{A B}^{x} f_{a b}^{x}}_{\text {epistasis }} & -\underbrace{\sum_{y=1}^{N} m^{y \rightarrow x} \frac{I^{y}}{I^{x}}\left(D^{x}-D^{y}-\left(f_{A}^{x}-f_{A}^{y}\right)\left(f_{B}^{x}-f_{B}^{y}\right)\right)}_{\text {migration }},
\end{aligned}
$$

where $s^{x}=f_{A}^{x} s_{A}^{x}+f_{B}^{x} s_{B}^{x}+f_{A B}^{x} s_{E}^{x}$ is the average selection for resistance, $f_{a b}^{x}=1-f_{A}^{x}-f_{B}^{x}+f_{A B}^{x}$ is the frequency of doubly-sensitive infections, and $\mu^{x}$ and $\rho^{x}$ are the total per-capita rates of mutation and recombination, respectively (e.g., $\mu^{x}=\mu_{a}^{x}+\mu_{A}^{x}+\mu_{b}^{x}+\mu_{B}^{x}$; Sup. Mat. 1 .

Equation (4) is partitioned into four key processes. First, excess selection for resistance to $\operatorname{drug} A$ (resp. $B), s_{A}^{x}-s^{x}$, can cause pre-existing LD $\left(D^{x} \neq 0\right)$ to increase or decrease. For example, if $s_{A}^{x}>s^{x}$ and $D^{x}>0$ then LD will increase. This is because drug $A$ resistant infections are fitter than the average resistant infection and so will increase in frequency. If $D^{x}>0$, it is more likely this increase will occur in doubly-resistant infections, thereby increasing $D^{x}$. Second, mutation and recombination removes any LD present at a rate proportional to the LD (Rice 2004: Slatkin 2008). Third, epistasis generates same-sign LD, that is, positive epistasis, $s_{E}^{x}>0$, leads to MDR over-representation, $D^{x}>0$ (Felsenstein 1965; Lewontin 1964a b; Lewontin and Kojima 1960). Positive epistasis could occur if double-resistance costs are less than expected 
(Hall and MacLean 2011; MacLean et al.2010; Trindade et al. 2009) or drugs are prescribed in combination (Bretscher et al. 2004; Day and Gandon 2012).

Migration is the final term of equation (4) and reveals how the metapopulation structure affects population LD. Like epistasis, migration does not require preexisting LD to operate on LD (Feldman and Christiansen 1975; Li and Nei 1974: Ohta|1982a b: Slatkin|1975). In particular, LD in population $x$ will be generated whenever the frequencies of resistance to drugs $A$ and $B$ differ between population $x$ and any other connected population, say $y$. If both types of resistance are more common in one population than the other, $\left(f_{A}^{x}-f_{A}^{y}\right)\left(f_{B}^{x}-f_{B}^{y}\right)>0$, then migration will generate positive LD in both populations, $D^{x}>0$ and $D^{y}>0$. If instead drug $A$ resistance is more prevalent in one population, while $\operatorname{drug} B$ resistance is more prevalent in the other, migration will generate negative LD in both populations.

Notice the presence of the multiplier $I^{y} / I^{x}$ in the final term of equation (4). If the populations have roughly the same density of infections, then this term is unimportant. However, when one population, say $y$, has much fewer total infections than population $x, I^{y} \ll I^{x}$, the term $I^{x} / I^{y}$ will be very large whereas $I^{y} / I^{x}$ will be very small. Consequently, the ability of migration to propagate LD will be greater in population $y$ than $x$, and so all else being equal we would predict the population with a lower density of infections will have a greater magnitude of LD than the population with a higher density of infections.

The next insight shows the importance of also taking into account equation (3). In particular, if we only inspected the migration term of equation (4) we might conclude that as the per-capita migration rate, $m^{y \rightarrow x}$, increases, so too will the ability of migration to propagate LD. However, the magnitude of population LD is actually maximized at intermediate migration rates (Fig. 2). The reason is because the quantity $m^{y \rightarrow x}$ has two effects. On the one hand, it directly multiplies the migration term in equation (4) thereby magnifying migration's potential role in LD build-up, while on the other hand, it also balances infection frequencies between populations (equation (3)), which in turn will reduce the magnitude of $\left(f_{A}^{x}-f_{A}^{y}\right)\left(f_{B}^{x}-f_{B}^{y}\right)$ in equation (4). These conflicting forces mean the magnitude of population LD tends to be maximized when migration is neither too infrequent nor too frequent (Fig. 2).

\section{Metapopulation-level multidrug resistance}

Now what happens to LD and MDR evolution at the metapopulation-level? Here we will use $\bar{X}$ to denote the metapopulation average of quantity $X^{x}$, e.g., $\bar{f}_{A}$ is the average $\operatorname{drug} A$ resistance in the metapopulation (see Sup. Mat. 3 for further details). Using this notation, then analogously to the population case, metapopulation LD is defined as $D_{\mathrm{M}} \equiv \bar{f}_{A B}-\bar{f}_{A} \bar{f}_{B}$. A more informative, but mathematically equivalent, description of metapopulation LD, however, is to define it in terms of the population variables as

$$
D_{\mathrm{M}} \equiv \bar{D}+\operatorname{cov}\left(f_{A}, f_{B}\right)
$$

that is, $D_{\mathrm{M}}$ is the sum of the average population $\mathrm{LD}, \bar{D}$, and the spatial covariance between the frequencies of resistance to drugs $A$ and $B$. Equation (5) shows that even if there is no population LD, i.e., $D^{x}=0$ and so $\bar{D}=0$, there still may be metapopulation LD; likewise, there may be population LD, $D^{x} \neq 0$, but no metapopulation LD, $D_{\mathrm{M}}=0$ (Feldman and Christiansen 1975; Nei and Li 1973; Ohta 1982a b).

With this in mind, the change in frequency of infections resistant to drug A (mutatis mu- 


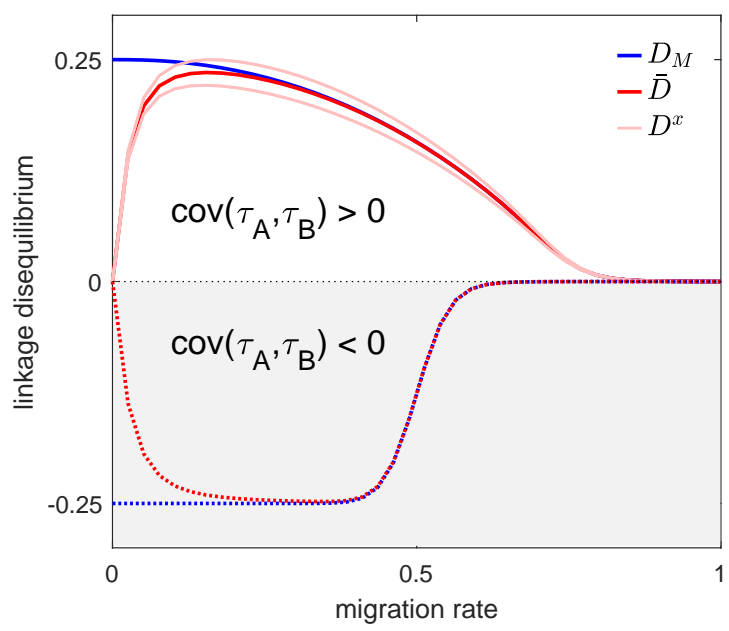

Figure 2: The effect of migration upon LD at equilibrium depends upon the scale at which LD is measured. Here we show equilibrium LD in a metapopulation consisting of four populations. Two scenarios are shown. In the first scenario (solid lines), $\operatorname{drug} A$ and $\operatorname{drug} B$ are both prescribed in the same two populations while the other two populations receive no drugs, thus $\operatorname{cov}\left(\tau_{A}, \tau_{B}\right)>0$; this yields $\operatorname{cov}\left(f_{A}, f_{B}\right)>0$ and so positive population, average, and metapopulation LD, i.e., $D^{x}, \bar{D}, D_{\mathrm{M}}>0$. In the second scenario (dashed lines), $\operatorname{drug} A$ is prescribed in two populations and $\operatorname{drug} B$ is prescribed in the other two populations, thus $\operatorname{cov}\left(\tau_{A}, \tau_{B}\right)<0$; this yields $\operatorname{cov}\left(f_{A}, f_{B}\right)<0$ and negative population, average, and metapopulation LD, i.e., $D^{x}, \bar{D}, D_{\mathrm{M}}<0$. Because we assume identical treatment rates and costs of resistance for either drug, in the second scenario all the populations have the same LD, whereas in the first scenario, since the drugs are prescribed unequally across populations, the LD observed in each of the two pairs of populations diverge. Specifically, populations experiencing greater selection due to increased drug prescription also have greater LD; this follows from the first term in equation (4).

tandis drug $B$ ) can be written

$$
\begin{aligned}
& \frac{\mathrm{d} \bar{f}_{A}}{\mathrm{~d} t}=\overbrace{\bar{s}_{A} \bar{f}_{A}\left(1-\bar{f}_{A}\right)}^{\begin{array}{c}
\text { direct } \\
\text { selection }
\end{array}}+\overbrace{\bar{s}_{B} D_{\mathrm{M}}}^{\text {indirect }}+\overbrace{\bar{s}_{E} \bar{f}_{A}\left(1-\bar{f}_{A}\right) \frac{\bar{f}_{A B}}{\bar{f}_{A}}}^{\text {epistasis }} \\
& +\underbrace{\left(\bar{\mu}_{A}+\bar{\rho}_{A}\right)\left(1-\bar{f}_{A}\right)-\left(\bar{\mu}_{a}+\bar{\rho}_{a}\right) \bar{f}_{A}}_{\text {mutation and recombination }}+\underbrace{\operatorname{cov}\left(r, f_{A}\right)}_{\begin{array}{c}
\text { heterogeneity in } \\
\text { 'baseline' growth }
\end{array}}+\underbrace{\bar{f}_{B} \operatorname{cov}\left(s_{B}, \frac{f_{A B}}{f_{B}}\right)}_{\begin{array}{c}
\text { heterogeneity in } \\
\text { indirect selection }
\end{array}} .
\end{aligned}
$$

The first four terms in equation (6) are the metapopulation-level analogues of the first four terms in equation (3); since they share the same interpretation, we do not discuss them further here. The last two terms, however, arise due to spatial heterogeneity in 'baseline' growth and selection and so are the consequence of population structure. As these terms are zero in the absence of spatial heterogeneities, they will be our focus here.

First, spatial heterogeneity arises through differences in the 'baseline' per-capita growth (i.e., $r^{x} \neq r^{y}$ ) coupled with differences in the frequencies of $\operatorname{drug} A$ resistant infections (i.e., 
$\left.f_{A}^{x} \neq f_{A}^{y}\right)$. This is the spatial covariance between 'baseline' per-capita growth and the frequency of drug $A$ resistant infections, $\operatorname{cov}\left(r, f_{A}\right)$. In particular, more productive (larger $r^{x}$ ) populations will have a disproportionate effect on the change in drug $A$ resistance. For example, if more productive populations also have a greater frequency of drug $A$ resistance, then heterogeneity increases the population frequency of $\operatorname{drug} A$ resistance. Heterogeneity in baseline growth could arise through a variety of mechanisms, such as availability of susceptible hosts, treatment rates differences, or pathogen traits (e.g., transmissibility and duration of carriage).

Second, spatial heterogeneity arises through differences in indirect selection for resistance to drug $B$ (i.e., $s_{B}^{x} \neq s_{B}^{y}$ ) coupled with differences in the probability that drug $B$ resistant infections are also doubly-resistant (i.e., $f_{A B}^{x} / f_{B}^{x} \neq f_{A B}^{y} / f_{B}^{y}$ ). This is the spatial covariance between selection on resistance to drug $B$ and the conditional probability that a drug $B$ resistant infection is doubly-resistant, $\operatorname{cov}\left(s_{B}, f_{A B} / f_{B}\right)$. In particular, populations experiencing greater selection for resistance to one drug will have a disproportionate effect on the change in frequency of infections resistant to the other drug, whenever populations differ in frequency of doublyresistant infections. As an example, if populations experiencing stronger selection for drug $B$ resistance also have a greater probability of drug $B$-resistant infections being doubly-resistant, heterogeneity in indirect selection increases the frequency of drug $A$ resistance in the metapopulation.

Next, the dynamics of metapopulation LD can be written as

$$
\begin{aligned}
\frac{\mathrm{d} D_{\mathrm{M}}}{\mathrm{d} t}=\overbrace{\left(\bar{s}_{A}-\bar{s}+\bar{s}_{B}-\bar{s}\right) D_{\mathrm{M}}}^{\text {selection }}-\overbrace{(\bar{\mu}+\bar{\rho}) D_{\mathrm{M}}}^{\text {mutation and }}+\overbrace{\bar{s}_{E} \bar{f}_{a b} \bar{f}_{A B}}^{\text {epistasis }} \\
+\underbrace{\operatorname{cov}(r, D)+\operatorname{coskew}\left(r, f_{A}, f_{B}\right)}_{\text {heterogeneity in 'baseline' growth }}+\sum_{d \in\{A, B\}}^{\sum_{\text {heterogeneity in resistance selection }}\left(1-\bar{f}_{d}\right) \bar{f}_{d} \operatorname{cov}\left(s_{d}, \frac{f_{A B}}{f_{d}}\right)},
\end{aligned}
$$

where coskew $\left(r, f_{A}, f_{B}\right)$ is the spatial coskewness between $r, f_{A}$, and $f_{B}$ and we have assumed population differences in mutation and recombination are negligible (see Sup. Mat. 3). The first three terms in equation (7) are the metapopulation level analogues of the first three terms of equation (4) and so share the same interpretation. The last two terms, however, arise due to spatial heterogeneity in 'baseline' growth and selection and so will be our focus here.

First, spatial heterogeneity arises through spatial differences in the 'baseline' per-capita growth (i.e., $r^{x} \neq r^{y}$ ) coupled with spatial heterogeneities in LD (i.e., $D^{x} \neq D^{y}$ ) or resistance frequencies (the coskewness term). The logic of the first term is clear: when population LD differs, more productive populations will disproportionately contribute to metapopulation LD. For the second term, when populations covary in frequency of resistance to $\operatorname{drug} A$ and $B$, more productive populations will disproportionately contribute to the covariance, $\operatorname{cov}\left(f_{A}, f_{B}\right)$ and so disproportionately contribute to metapopulation LD (through the second term in equation (5)).

Second, spatial heterogeneity arises through differences in selection for resistance $\left(s_{d}^{x} \neq\right.$ $s_{d}^{y}$ ) coupled with differences in the proportion of drug $d$ resistant infections that are doublyresistant $\left(f_{A B}^{x} / f_{d}^{x} \neq f_{A B}^{y} / f_{d}^{y}\right)$. The logic here is that populations experiencing stronger selection for resistance are more likely to see an increase in resistant infections. If this increase occurs disproportionately in doubly-resistant infections, then from equation (1) metapopulation LD will increase, whereas if this increase occurs disproportionately in singly-resistant infections, metapopulation LD will decrease. The magnitude of this effect is scaled by $\bar{f}_{d}\left(1-\bar{f}_{d}\right)$ 
since selection cannot operate without genetic variation. In the absence of population LD, then $f_{A B}^{x} / f_{A}^{x}=f_{B}^{x}$ and $f_{A B}^{x} / f_{B}^{x}=f_{A}^{x}$, and so if populations experiencing stronger selection for resistance to one drug also have a greater frequency of infections resistant to the other drug, metapopulation LD will increase. This could occur if, for example, some populations experience greater treatment rates.

As a final note, observe that in contrast to equation (4), in equation (7) the per-capita migration rates $m^{y \rightarrow x}$ are nowhere to be found. The reason for this is intuitive: as migration does not affect the total density of infecteds, nor the resistance status of an infection, it will not change the quantities $\bar{f}_{A B}, \bar{f}_{A}$, or $\bar{f}_{B}$, and so cannot change metapopulation LD. As a consequence, migration only affects metapopulation LD indirectly by reducing differences in infection frequency between populations, thereby dampening the magnitude (and hence the effect) of $\operatorname{cov}(r, D), \operatorname{cov}\left(r, f_{d}\right)$, and $\operatorname{cov}\left(s_{\ell}, f_{A B} / f_{d}\right)$ in equation (7). It follows that, all else being equal, the magnitude of $D_{\mathrm{M}}$ is a decreasing function of the per-capita migration rate, and so is maximized when migration is infrequent (Fig. 2).

\section{Modeling the dynamics of LD: why bother?}

To this point we have focused upon developing the LD perspective to provide a conceptual understanding of MDR evolution in structured populations. However, framing the LD perspective in terms of general quantities has meant this conceptual understanding is somewhat abstract. What we now wish to demonstrate, through the consideration of three scenarios, is how the LD perspective can be used to tackle practical problems. In the first scenario, we show how the LD perspective helps us generalize a recent paper on the effect of spatial structure on equilibrium patterns of MDR. In the second scenario, we show how the LD perspective allows for an understanding of transient dynamics, and apply this insight to patterns of MDR observed in Streptococcus pneumoniae. In the third scenario we show how the LD perspective generates practical insight into designing drug prescription strategies across populations, with a focus upon a hospital-community setting.

\section{LD perspective explains equilibrium patterns of MDR}

Understanding the patterns of MDR in structured populations was first tackled in an important paper by (Lehtinen et al.|2019). The paper by Lehtinen et al. (2019) (see also Jacopin et al. 2020) focused upon MDR evolution in a metapopulation consisting of independent host populations (so migration is restricted, $m^{x \rightarrow y} \approx 0$ ). For example, each population could represent a different Streptococcus pneumoniae serotype maintained by serotype-specific host immunity (Cobey and Lipsitch 2012; Henriques-Normark and Tuomanen|2013; Lehtinen et al. 2017, 2019). Lehtinen et al. (2019) found that at equilibrium, population differences could lead to MDR overrepresentation $\left(D_{\mathrm{M}}>0\right)$, and that populations with a longer duration of pathogen carriage were more likely to exhibit MDR, a result they attributed to an increased likelihood of antibiotic exposure. Here we show how employing the LD perspective: (i) reveals the evolutionary logic behind what populations differences maintain metapopulation LD at equilibrium, and (ii) using these insights allows us to generalize the results to a broader range of scenarios, beyond variation in duration of carriage. For simplicity, we will assume there is no epistasis (i.e. $s_{E}^{x}=0$ ).

To maintain metapopulation $\mathrm{LD}$ at equilibrium, there needs to be at minimum some mechanism maintaining metapopulation resistance diversity (i.e., $0<\bar{f}_{d}<1$ ), otherwise $D_{\mathrm{M}}=0$. 
There are variety of ways in which this could occur (Colijn et al.2009; Davies et al.2019; Jacopin et al. 2020; Krieger et al. 2020; Lehtinen et al. 2017, 2019; Lipsitch et al.2009), but Lehtinen et al. (2017,2019) assume it is due to some variation among populations in the conditions favouring resistance evolution. This mechanism maintains diversity at the scale of the metapopulation but leads to the fixation or the extinction of drug resistance locally. Thus $D^{x}=0$, and it follows from equation (5) that $D_{\mathrm{M}}=\operatorname{cov}\left(f_{A}, f_{B}\right)$. Therefore, in order for metapopulation LD to exist, $f_{A}$ and $f_{B}$ must covary. Specifically, whenever $f_{A}^{x}$ and $f_{B}^{x}$ (or their dynamical equations, (3)), are uncorrelated, the metapopulation will be in linkage equilibrium. From equation (3) we see that if the additive selection coefficients, $s_{A}^{x}$ and $s_{B}^{x}$, are uncorrelated, then so too are the dynamics of $f_{A}^{x}$ and $f_{B}^{x}$, and $\operatorname{so} \operatorname{cov}\left(f_{A}, f_{B}\right)=0$. Hence only when population differences generate correlations between the selection coefficients will they generate LD.

Using this insight, why are populations with a longer duration of carriage associated with MDR? And should we expect associations between MDR and any other population attributes? Our primary focus is whether (and how) the selection coefficients are correlated. Letting $\Delta z_{k}^{x}$ be the contribution of trait $z$ to the additive selection coefficient for resistance to drug $d$ in population $x$ (e.g., $\Delta \beta_{A}^{x}=\beta_{A b}^{x}-\beta_{a b}^{x}$ ), then it is straightforward to compute (see Sup. Mat. 4.1),

$$
\begin{gathered}
s_{A}^{x}=\Delta \beta_{A}^{x} S^{x}-\Delta \alpha_{A}^{x}+\tau_{A}^{x}, \\
s_{B}^{x}=\Delta \beta_{B}^{x} S^{x}-\Delta \alpha_{B}^{x}+\tau_{B}^{x} .
\end{gathered}
$$

where we have used slightly different notation from Lehtinen et al. (2019). Now, consider a scenario in which both the treatment rates and the parameters controlling the (additive) costs of resistance are uncorrelated (i.e., $\Delta \beta_{d}^{x}=\Delta \beta_{d}, \Delta \alpha_{d}^{x}=\Delta \alpha_{d}$ and $\tau_{d}^{x}=\tau_{d}$; this is one of the scenarios presented in Figure 4 of Lehtinen et al. (2019)). From equation (8), the only remaining source of correlation is susceptible density, $S^{x}$, which plays a role whenever there are explicit transmission costs, $\Delta \beta_{d}<0$. At equilibrium, $S^{x}$ is determined by pathogen traits such as transmission and duration of carriage, such that 'fitter' populations (i.e., those in which pathogens are more transmissible or have longer duration of carriage) will more substantially deplete susceptibles. By reducing $S^{x}$, 'fitter' populations lower the transmission costs for resistance to either drug, and so double-resistance is more likely to be selectively advantageous, even when treatment rates are uncorrelated. In turn, this over-representation of doubly-resistant infections will generate metapopulation LD.

Thus although variation in duration of carriage can lead to MDR evolution and LD through its effect upon susceptible density (Fig. 3a), it is neither necessary (the same pattern can be produced by variation in transmissibility; Fig. $3 \mathrm{~b}$ ) nor sufficient (variation in duration of carriage has no effect without explicit transmission costs, Fig. $3 \mathrm{c}$ ). More broadly, if there are more than two drugs, then provided that there are explicit transmission costs for resistance to each drug, susceptible density will generate a correlation between all the selection coefficients, which in turn will yield the pattern of 'nestedness' observed by Lehtinen et al. (2019). What is critical for this effect to be prominent, however, is that there is clear differentiation in population susceptible density, and that the parameters controlling cost of resistance (i.e., $\Delta \beta_{d}$ ), are large enough so as to ensure a strong correlation amongst selection coefficients.

\section{LD perspective explains transient patterns of MDR}

The predictions of Lehtinen et al. (2019) were used to explain the patterns of MDR observed in surveillance data. One of these data sets was a surveillance study that documented both the 

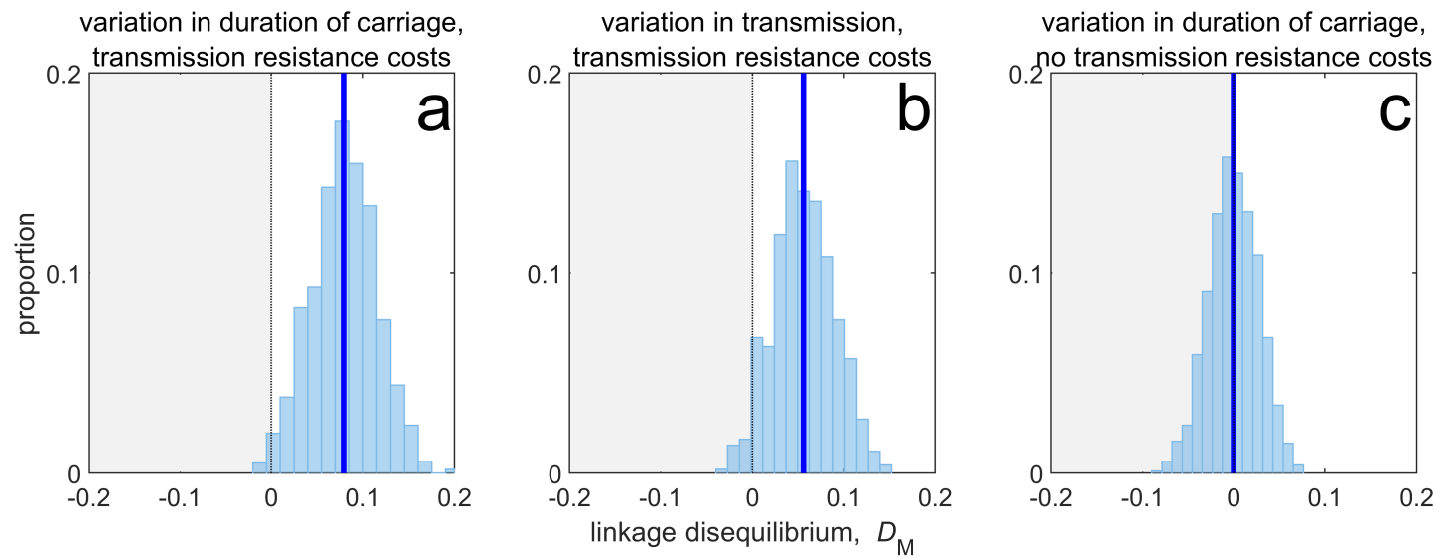

Figure 3: Duration of carriage is one of many potential explanations for MDR overrepresentation at equilibrium. Variation in duration of carriage across independent populations can lead to linkage disequilibrium (subplot a), but it is neither necessary (b), nor sufficient (c). We simulate 1000 populations (blue bars), each consisting of 20 independent populations in which treatment rates for each population are randomly chosen to be either $\tau_{\max }=0.075$ or $\tau_{\min }=0.025$ with equal probability while simultaneously satisfying $\operatorname{cov}\left(\tau_{A}, \tau_{B}\right)=0$. The solid blue line is the mean LD across the simulations for each scenario. In subplot a, duration of carriage varies across populations and there are transmission resistance costs; in subplot $\mathbf{b}$, transmission varies and there are transmission resistance costs; while in subplot c, duration of carriage varies and there are no transmission costs. These simulations diverge slightly from those of Lehtinen et al. (2019) in that their model always includes epistasis (see Sup. Mat. 4.1), whereas here we only consider non-epistatic scenarios.

serotype as well as antibiotic resistance to a number of different drugs in S. pneumoniae infections sampled in Maela, Northern Thailand (Lehtinen et al.|2019: Turner et al.2012). Although the prediction of positive (metapopulation) LD was met for most drug combinations (Lehtinen et al. 2019), inspection of the data set reveals significant serotype LD (Fig. 4). This is notable because, as we have detailed above, at equilibrium the model of Lehtinen et al. (2019) predicts each serotype will be in linkage equilibrium, $D^{x}=0$. How can we reconcile these conflicting observations? Although there are various possible explanations (e.g., an additional mechanism capable of maintaining diversity within-serotype), here we focus upon relaxing the assumption that the metapopulation is at equilibrium. That is, we are interested in whether long term transient dynamics unfolding over months and years can plausibly explain the observed serotype LD.

To do so, consider a metapopulation consisting of independent serotypes, differing in their transmissibility and duration of carriage (as in the model of Lehtinen et al. (2017, 2019)). Assume that there is no epistasis and that the additive selection coefficients take the form of equation (8), where the parameters $\Delta \beta_{d}^{x}, \Delta \alpha_{d}^{x}$ and $\tau_{d}^{x}$ do not depend upon serotype $x$ (Sup. Mat. 4.2. Suppose that initially the metapopulation is treated exclusively with $\operatorname{drug} A$ at sufficiently high rates such that resistance to $\operatorname{drug} A$ goes to fixation in each serotype, i.e., $\bar{f}_{A} \rightarrow 1$ and $\bar{f}_{B} \rightarrow 0$, and so $D_{\mathrm{M}}=0$. Now suppose at time $t=1000$ months that drug $B$ is 'discovered' and subsequently prescribed at a high rate in the metapopulation, while owing to its reduced efficacy, prescription 


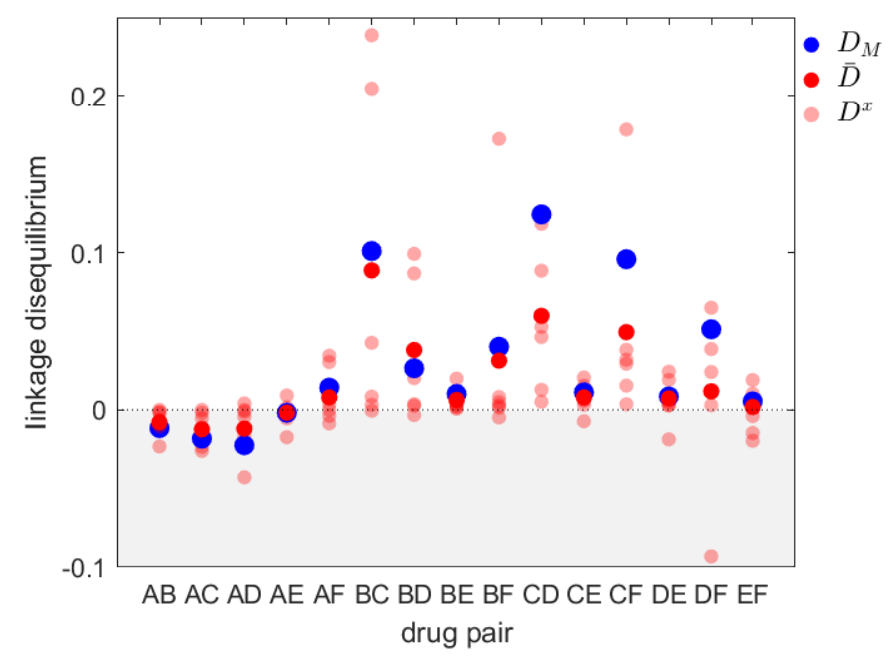

Figure 4: Linkage disequilibrium for different drug pairs in Streptococcus pneumoniae. Data is from the Maela surveillance data set of Lehtinen et al. (2019); Turner et al. (2012). The light red circles are the observed serotype $\mathrm{LD}, D^{x}$, the dark red circles are the average LD across serotypes, $\bar{D}$, while the blue circles are the metapopulation $\mathrm{LD}, D_{\mathrm{M}}$. We have restricted the data to serotypes involving 100 or more samples (serotypes 14, 6A/C, 6B, 15B/C, 19F, 23F). The drugs considered are: $\mathrm{A}=$ chloramphenicol, $\mathrm{B}=$ clindamycin, $\mathrm{C}=$ erythromycin, $\mathrm{D}=$ penicillin, $\mathrm{E}=$ sulphatrimethoprim, and $\mathrm{F}=$ tetracycline.

of $\operatorname{drug} A$ is reduced. Although the treatment rates do not vary by serotype, serotype differences in transmissibility and duration of carriage mean that the changes to treatment rates will differentially affect serotype density, which in turn will differentially affect the serotype-specific availability of susceptible hosts, $S^{x}$. Since the serotype-specific selection coefficients, $s_{A}^{x}$ and $s_{B}^{x}$, and baseline per-capita growth, $r^{x}$, directly depend upon $S^{x}$, the variation in $S^{x}$ introduces heterogeneity in equation (7), which in turn generates metapopulation LD. Because the selection coefficients are positively correlated, the metapopulation LD generated will be positive, i.e., $D_{\mathrm{M}}>0$ (Fig. 5a,d) From equation (7), once metapopulation LD is generated, it will be amplified by directional selection (first term of (7)) which is initially positive since resistance to drug $B$ is favoured; this leads to a rapid build up of $D_{\mathrm{M}}$ (Fig. $5 \mathbf{a}$,d). However, this initial increase in $D_{\mathrm{M}}$ is transient; for this particular choice of parameter values, at equilibrium $D_{\mathrm{M}} \rightarrow 0$. Crucially, however, the changes to $D_{\mathrm{M}}$ can unfold over a very long time (here the time units are months), such that surveillance data would detect little change in the metapopulation dynamics and so suggest a population roughly in equilibrium.

Although this scenario can lead to considerable (transient) metapopulation LD, there is still nothing generating serotype LD. Our analysis of equation (4) revealed two possible (deterministic) mechanisms capable of generating population (serotype) LD. First, migration between populations can lead to metapopulation LD spilling over into population LD. In this example, 'migration' between serotypes would correspond to serotype 'switching' (Croucher et al.|2015), whereby infections exchange serotypes through recombination. However, this is an unlikely explanation as the rate of serotype switching would have to be unrealistically large for serotype LD to be substantially altered. The second term in equation (4) capable of generating serotype 

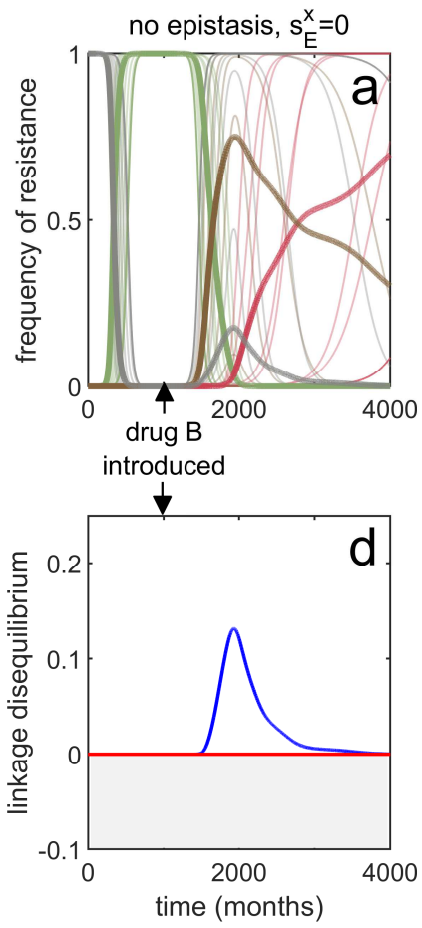
(Fig. 5e).

LD is epistasis, which will generate same sign serotype LD (Felsenstein 1965 ; Lewontin $1964 \mathrm{a}$ b; Lewontin and Kojima 1960). Indeed, in the model considered, negative epistasis, $s_{E}^{x}<0$, generates transient negative serotype LD (Fig. 5b,e), while positive epistasis generates transient positive serotype LD (Fig 5c,f; Sup. Mat. 4.2). Notably, although negative epistasis produces negative serotype LD (and so $\bar{D}<0$ ), at the scale of the metapopulation this effect is swamped by the positive covariance in frequency of resistance and so metapopulation LD is positive, $D_{\mathrm{M}}>0$.
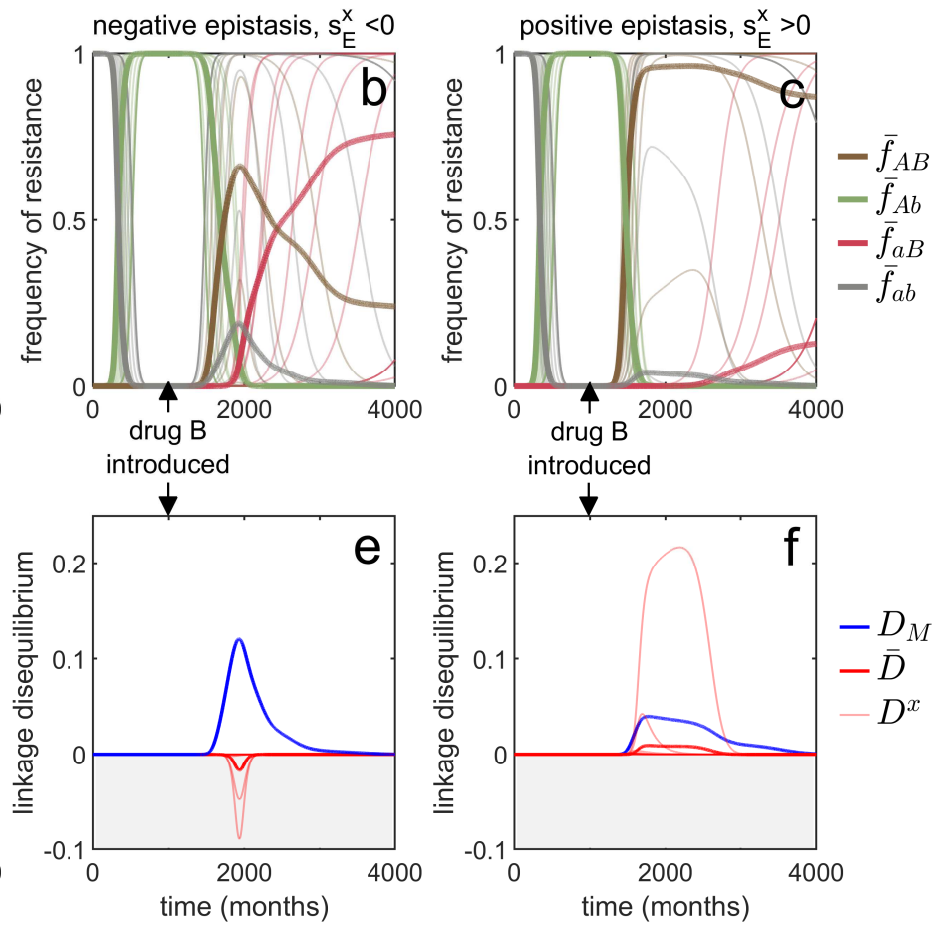

Figure 5: Transient dynamics coupled with epistasis can explain patterns of serotype LD in Streptococcus pneumoniae. In all simulations, serotypes differ based upon duration of carriage and transmissibility. At $t=0$, the pathogen is sensitive to both drugs; however as hosts are initially treated with drug $A$ at a rate of $\tau_{A}=0.12$ per month, resistance to drug $A$ emerges and fixes in all serotypes. At $t=1000$ (months), $\operatorname{drug} B$ is introduced, and $\operatorname{drug} A$ prescription reduced, $\left(\tau_{A}, \tau_{B}\right)=(0.07,0.1)$ (note that the drugs are never prescribed in combination, $\left.\tau_{A B}=0\right)$. In the first column, there is no epistasis, thus although metapopulation LD builds up, serotype LD does not. In the second column, there is negative epistasis, which generates negative serotype LD. In the third column, there is positive epistasis which produces positive serotype LD. The thin lines denote the within-serotype dynamics, while the thick lines denote the metapopulation dynamics. In all cases, at equilibrium both the serotypes and the metapopulation will be in linkage equilibrium, however, transient LD can occur on sufficiently long timescales so as to appear permanent (see Sup. Mat. 4.2 for more details).

Thus transient dynamics coupled with epistasis could provide a potential explanation for the significant within-serotype LD observed in S. pneumoniae. More generally, the potential complexity of competing selective pressures associated with multilocus dynamics can lead to prolonged, but transient, polymorphisms and LD, and so surveillance data showing limited 
temporal change in resistance frequency should be treated cautiously and not assumed to be due to a stable equilibrium.

\section{LD perspective helps identify drug prescription strategies limiting the evolu- tion of MDR}

Understanding the evolutionary consequences of different antibiotic prescription strategies across populations can have practical relevance for public health. The populations of interest could correspond to physically distinct groups such as a hospital and its broader community, or different geographical regions (e.g., countries). From a public health perspective, when considering different prescription strategies, a variety of factors must be considered, but in general the goal is to successfully treat as many people as possible, thereby reducing the total burden (Bonhoeffer et al. 1997: zur Wiesch et al.2014). In this circumstance, the LD in the metapopulation and/or populations can provide important information about the likelihood of treatment success. In particular, for a given population frequency of drug $A$ and drug $B$ resistance, negative LD (MDR under-representation) increases the likelihood that if treatment with one drug fails (due to resistance), treatment with the other drug will succeed. On the other hand, positive LD (MDR over-representation) increases the likelihood of treatment failure, since a greater proportion of resistant infections are doubly-resistant and so cannot be successfully treated with either drug. Equations (4) and (7) show that to generate negative LD, drugs should be deployed in a population specific fashion, that is, drug $A$ should be restricted to some populations and drug $B$ restricted to the remaining populations (see also Day and Gandon 2012; Jacopin et al. 2020 Lehtinen et al. 2019). Doing so will create a negative covariance in selection, such that resistance to drug $A$ (resp. drug $B$ ) will be favoured in some populations and disfavoured in the others. This negative covariance in selection will give rise to negative LD and MDR underrepresentation (Fig. 2).

As an application of this principle, consider two populations connected by migration, corresponding to a 'community' and a much smaller 'hospital'. Drug prescription occurs at a fixed (total) rate in each population, while the prescription rate is much higher in the hospital (see Sup. Mat. 4.3. Consider three antibiotic prescription strategies: (i) drugs can be randomly prescribed to individuals (mixing); (ii) drugs can be prescribed exclusively in combination; or (iii) prescription of drug $A$ and $B$ can be asynchronously rotated between the hospital and community, that is, if the hospital uses drug $A$ then the community uses drug $B$, and vice versa (cycling). As both drugs are prescribed at higher rates in the hospital than the community, both mixing and combination generate a positive covariance in selection across populations, producing positive LD and MDR over-representation (see equation (4); Fig. 6). Thus over the short- and long-term, mixing and combination produce similar results: doubly-resistant infections are favoured, while singly-resistant infections are disfavoured (Fig. 6). Now consider cycling. When drugs are rotated rapidly between populations, infections in either population are likely to be exposed to both drugs. Because prescription rates are higher in the hospital, this effectively creates a positive covariance in selection (i.e., cycling behaves like mixing) and so when resistance emerges, infections tend to be doubly-resistant (MDR over-representation; Fig. S1). When drugs are rotated less frequently, infections are more likely to be exposed to a single drug, creating a negative covariance in selection across populations. In this circumstance, although single resistance can emerge at lower treatment rates then when rotations are more frequent, the negative LD produced by the negative covariance in selection inhibits the 
emergence of double-resistance (MDR under-representation; Fig. 6).

These results emphasize an important trade-off: delaying the evolution of MDR (e.g., by decreasing time between rotations) promotes the evolution of single drug resistance, whereas delaying the evolution of single drug resistance promotes the evolution of MDR. This is logical: when we maintain a constant treatment rate per individual, decreasing selection for the 'generalist' strategy (MDR) necessarily increases selection for the 'specialist' strategy (single drug resistance) (Wilson and Yoshimura 1994). Thus cycling can either be the best, or worst, option for single drug resistance (Beardmore et al.2017); but critically, this has concomitant effects for MDR (see also Fig. 6). Indeed, mixing, combination and cycling have been exhaustively compared in the context of single drug resistance (e.g., Beardmore and Peña-Miller 2010; Beardmore et al. 2017; Bergstrom et al. 2004; Bonhoeffer et al. 1997; Lipsitch et al. 2000; Tepekule et al.|2017; zur Wiesch et al.2014); yet these studies largely ignored the consequences for MDR evolution. Our analysis suggests controlling for single drug resistance will have important consequences for MDR, and so it should not be considered in isolation. More generally, whether it is optimal to either delay single drug resistance or prevent MDR will depend upon what metric is used to evaluate what constitutes a 'success' or 'failure'.
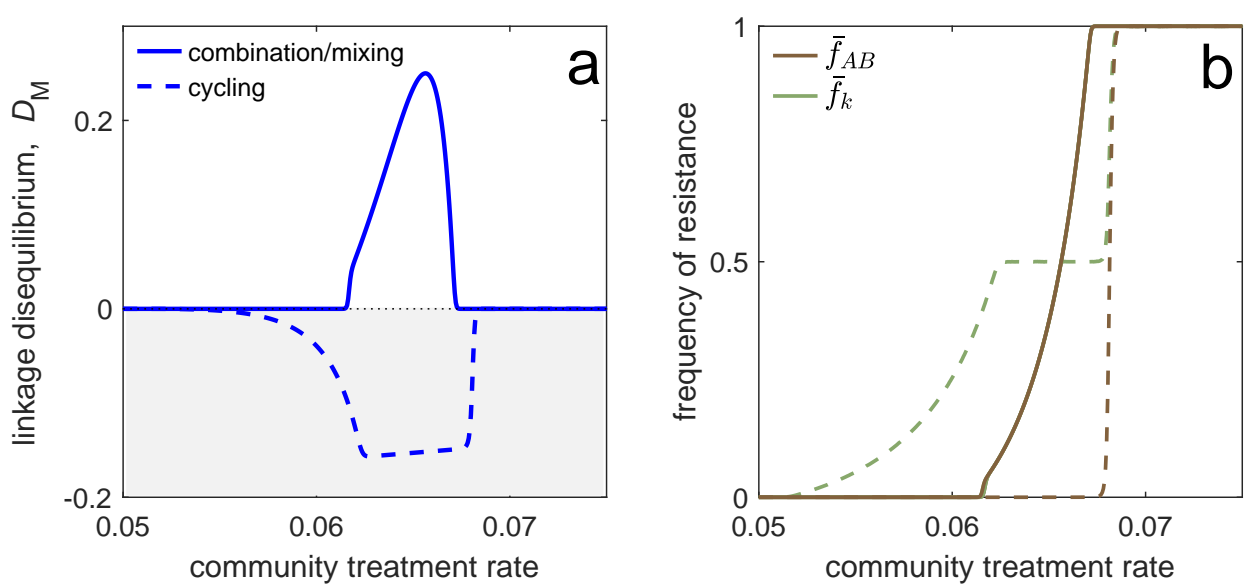

Figure 6: Different antibiotic prescription strategies generate different patterns of LD at equilibrium. Here we focus upon a population divided into a community and a hospital. Individuals enter the hospital at a fixed rate and spend a fifth of the time in the hospital that it takes to naturally clear a sensitive infection. The hospital/community size split corresponds to 20 beds per 1000 people, while individuals in the hospital receive antibiotics at 15x the rate they do in the community. We integrate system (2) until equilibrium is reached; the final state of the system is what is shown. For cycling, we compute the average state over the last two rotations (i.e., over the last period, $T$; in this case $T=100$ ). In panel a we show the metapopulation $L D$, $D_{\mathrm{M}}$ for the three treatment scenarios (combination, mixing, cycling). Combination and mixing generate identical LD in this example. In panel $\mathbf{b}$ we show the frequency of infections in the metapopulation resistant to drug $d, \bar{f}_{d}$ (for our choice of parameters, $\bar{f}_{A}=\bar{f}_{B}$; Sup. Mat. 4.3 , and doubly-resistant, $\bar{f}_{A B}$, for each scenario. Note that for mixing and combination treatments (solid curves), $\bar{f}_{A}=\bar{f}_{B}=\bar{f}_{A B}$, whereas cycling (dashed curves) leads to singly-resistant infections at low treatment rates (see Sup. Mat. 4.3p. 


\section{Conclusions}

The evolution of multidrug resistant pathogens is a pressing health concern, and is a topic which is increasingly gaining attention from evolutionary biologists and mathematical modellers alike. However, the typical process in studying the problem of MDR is to introduce a model of the form of (2), and then either proceed to a numerical analysis of these equations or simplify the model further by neglecting the dynamics of double resistant infections (Beardmore et al. 2017; Bergstrom et al. 2004; Bonhoeffer et al. 1997). This is because models of MDR evolution rapidly become intractable, a problem which is particularly acute when incorporating aspects of population structure. Here we have argued that a more insightful and simplifying approach is the 'linkage disequilibrium perspective': after specifying the model of interest, as in (2), it is desirable to transform the model into the form of equations (3), (4), (6), and (7), which brings to the forefront the role played by linkage disequilibrium for MDR evolution in structured populations.

Our analysis emphasizes that metapopulation structure alone can generate and maintain LD (and so MDR), even in the absence of epistasis (Li and Nei 1974; Nei and Li 1973; Ohta 1982a b; Slatkin 1975). Since in natural populations metapopulation structure is often hidden (e.g., Rosen et al. 2015), patterns of MDR should not be assumed to be due to epistasis, even if no structure is readily apparent. Moreover, caution must be taken when measuring LD (and MDR) at a particular scale, as doing so can lead to erroneous conclusions: even if the metapopulation is in linkage equilibrium, $D_{\mathrm{M}}=0$, the populations need not be, $D^{x} \neq 0$, and vice versa (equation (5)), while in more extreme cases, population and metapopulation LD can be of opposite sign (Fig. 5b,e). These are not merely esoteric points; the presence or absence of LD (and MDR), and its source (epistasis or metapopulation structure) is critically important. For example, when MDR is due to metapopulation structure rather than epistasis, prescribing drugs across different populations so as to create a negative covariance in selection can reduce the prevalence of MDR (Fig. 6; Day and Gandon (2012); Jacopin et al. (2020); Lehtinen et al. (2019)), while distinguishing between population and metapopulation LD can provide additional insight towards evaluating hypotheses (Fig. 4 and 5).

Our analysis assumed that the evolutionary dynamics were deterministic, thus neglecting the influence of stochasticity. However it is widely appreciated in population genetics that stochasticity can play an important role in multilocus dynamics. For example, LD can be generated through genetic drift (Barton 1995; Hill and Robertson 1966; Keightley and Otto 2006; Lenormand and Otto 2000; Martin et al.2006; Otto and Barton 2001), which in turn can interfere with the strength of selection (Hill and Robertson 1966; Neher and Shraiman 2011; Slatkin 2008). Similarly, the (random) genetic background a rare mutation finds itself upon is critically important for its success (Gillespie 2000; Kouyos et al. 2006; Neher 2013), and in finite populations this alone can generate LD. However, little has been done to relate these results to evolutionary epidemiology, or to understand how epidemiological feedbacks can influence their predictions. The little work to date has relied upon complex simulations (e.g., Althaus and Bonhoeffer 2005; Kouyos et al. 2009), which necessarily sacrifice general insight for specificity. Thus the role of stochasticity in the evolution of MDR remains an area in which further investigation is warranted.

Understanding the evolution of MDR is a research topic of pressing concern. Here we have argued that using the linkage disequilibrium perspective leaves us better equipped to determine what factors are responsible for generating MDR, and their generality. Moreover, taking 
bioRxiv preprint doi: https://doi.org/10.1101/2020.07.31.230896; this version posted December 10,2020 . The copyright holder for this preprint (which was not certified by peer review) is the author/funder, who has granted bioRxiv a license to display the preprint in perpetuity. It is made available under aCC-BY-NC-ND 4.0 International license.

438 such an approach leads to a more straightforward comparison with existing models and results. 


\section{References}

Althaus, C. I. and S. Bonhoeffer. 2005. Stochastic interplay between mutation and recombination during acquisition of drug resistance mutations in human immunodeficiency virus type 1. J. Virol. 79:13572-13578.

Austin, D. J. and R. M. Anderson. 1999. Studies of antibiotic resistance within the patient, hospitals and the community using simple mathematical models. Phil. Trans. R. Soc. B 354:721738.

Barton, N. H. 1995. Linkage and the limits to natural selection. Genetics 140:821-841.

Beardmore, R. E. and R. Peña-Miller. 2010. Antibiotic cycling versus mixing: the difficulty of using mathematical models to definitively quantify their relative merits. Math. Biosci. Eng. 7:923-933.

Beardmore, R. E., R. Peña-Miller, F. Gori, and J. Iredell. 2017. Antibiotic cycling and antibiotic mixing: which one best mitigates antibiotic resistance? Mol. Biol. Evol. 34:802-817.

Bergstrom, C. T., M. Lo, and M. Lipsitch. 2004. Ecological theory suggests that antimicrobial cycling will not reduce antimicrobial resistance in hospitals. PNAS 101:13285-13290.

Blanquart, F. 2019. Evolutionary epidemiology models to predict the dynamics of antibiotic resistance. Evol. Appl. 12:365-383.

Bonhoeffer, S., M. Lipsitch, and B. R. Levin. 1997. Evaluating treatment protocols to prevent antibiotic resistance. Proc. Natl. Acad. Sci. 94:12106-12111.

Bretscher, M. T., C. L. Althaus, V. Muller, and S. Bonhoeffer. 2004. Recombination in HIV and the evolution of drug resistance: for better or for worse? BioEssays 26:180-188.

Cobey, S. and M. Lipsitch. 2012. Niche and neutral effects of acquired immunity permit coexistence of pneumococcal serotypes. Science 335:1376-1380.

Colijn, C., T. Cohen, C. Fraser, W. Hanage, E. Goldstein, N. Givon-Lavi, R. Dagan, and M. Lipsitch. 2009. What is the mechanism for persistent coexistence of drug-susceptible and drugresistant strains of Streptococcus pneumoniae? J. R. Soc. Interface 7:905-919.

Croucher, N. J., L. Kagedan, C. M. Thompson, J. Parkhill, S. D. Bentley, J. A. Finkelstein, M. Lipsitch, and W. P. Hanage. 2015. Selective and genetic constraints on pneumococcal serotype switching. PLoS Genetics 11:e1005095.

Davies, N. G., S. Flasche, M. Jit, and K. E. Atkins. 2019. Within-host dynamics shape antibiotic resistance in commensal bacteria. Nat. Ecol. Evol. 3:440-449.

Day, T. and S. Gandon. 2012. The evolutionary epidemiology of multilocus drug resistance. Evolution 66:1582-1597.

Feldman, M. W. and F. B. Christiansen. 1975. The effect of population subdivision on two loci without selection. Genet. Res. Camb. 24:151-162. 
Felsenstein, J. 1965. The effect of linkage on directional selection. Genetics 52:349-363.

Fisher, R. A., 1930. The Genetical Theory of Natural Selection. Clarendon Press.

Gillespie, J. H. 2000. Genetic drift in an infinite population: the pseudohitchhiking model. Genetics 155:909-913.

Hall, A. R. and R. C. MacLean. 2011. Epistasis buffers the fitness effects of rifampicin-resistance mutations in Pseudomonas Aeruginosa. Evolution 65:2370-2379.

Henriques-Normark, B. and E. I. Tuomanen. 2013. The pneumococcus: epidemiology, microbiology, and pathogenesis. Cold Spring Harb. Perspect. Med. Pp. 1-15.

Hill, W. G. and A. Robertson. 1966. The effect of linkage on limits to artifical selection. Genet. Res. Camb. 8:269-294.

Jacopin, E., S. Lehtinen, F. Débarre, and F. Blanquart. 2020. Factors favouring the evolution of multidrug resistance in bacteria. J. Roy. Soc. Interface 17:1-14.

Keightley, P. D. and S. P. Otto. 2006. Interference among deleterious mutations favours sex and recombination in finite populations. Nature 443:89-92.

Kouyos, R. D., D. Fouchet, and S. Bonhoeffer. 2009. Recombination and drug resistance in HIV: population dynamics and stochasticity. Epidemics 1:58-69.

Kouyos, R. D., S. P. Otto, and S. Bonhoeffer. 2006. Effect of varying epistasis on the evolution of recombination. Genetics 173:589-597.

Krieger, M. S., C. E. Denison, T. L. Anderson, M. A. Nowak, and A. L. Hill. 2020. Population structure across scales facilitates coexistence and spatial heterogeneity of antibiotic-resistant infections. PLoS Comp. Biol. 16:e1008010.

Lehtinen, S., F. Blanquart, N. J. Croucher, P. Turner, M. Lipsitch, and C. Fraser. 2017. Evolution of antibiotic resistance is linked to any genetic mechanism affecting bacterial duration of carriage. Proc. Natl. Acad. Sci. 114:1075-1080.

Lehtinen, S., F. Blanquart, M. Lipsitch, and C. Fraser. 2019. On the evolutionary ecology of multidrug resistance in bacteria. PLoS Pathogens .

Lenormand, T. and S. P. Otto. 2000. The evolution of recombination in a heterogeneous environment. Genetics 156:423-438.

Lewontin, R. C. 1964a. The interaction of selection and linkage I: General conditions; heterotic models. Genetics 49:49-67. 782.

Lewontin, R. C. and K. Kojima. 1960. The evolutionary dynamics of complex polymorphisms. Evolution 14:458-472. 
Li, W. and M. Nei. 1974. Stable linkage disequilibrium without epistasis in subdivided populations. Theor. Pop. Biol. 6:173-183.

Lipsitch, M., C. T. Bergstrom, and B. R. Levin. 2000. The epidemiology of antibiotic resistance in hospitals: paradoxes and prescriptions. PNAS 97:1938-1943.

Lipsitch, M., C. Colijn, T. Cohen, W. P. Hanage, and C. Fraser. 2009. No coexistence for free: neutral null models for multistrain pathogens. Epidemics 1:2-13.

MacLean, R. C., A. R. Hall, G. G. Perron, and A. Buckling. 2010. The population genetics of antibiotic resistance: integrating molecular mechanisms and treatment contexts. Nature Reviews Genetics 11:405-414.

Martin, G., S. P. Otto, and T. Lenormand. 2006. Selection for recombination in structured populations. Genetics 172:593-609.

Neher, R. A. 2013. Genetic draft, selective interference, and population genetics of rapid adaptation. Annu. Rev. Ecol. Evol. Syst. 44:195-215.

Neher, R. A. and B. I. Shraiman. 2011. Statistical genetics and evolution of quantitative traits. Rev. Mod. Phys. 83:1283-1300.

Nei, M. and W. Li. 1973. Linkage disequilibrium in subdivided populations. Genetics 75:213219.

Ohta, T. 1982a. Linkage disequilibrium due to random genetic drift in finite subdivided populations. Proc. Natl. Acad. Sci. 79:1940-1944.

1982b. Linkage disequilibrium with the island model. Genetics 101:139-155.

O’Neill, J. 2015. Tackling a global health crisis: initial steps. The Review on Antimicrobial Resistance Chaired by Jim O’Neill .

Otto, S. P. and N. H. Barton. 2001. Selection for recombination in small populations. Evolution 55:1921-1931.

Rice, S. H., 2004. Evolutionary Theory: Mathematical and Conceptual Foundations. Sinauer Associates, Sunderland, MA, USA.

Rosen, M. J., M. Davison, D. Bhaya, and D. S. Fisher. 2015. Fine-scale diversity and extensive recombination in a quasisexual bacterial population occupying a broad niche. Science 348:1019-1023.

Slatkin, M. 1975. Gene flow and selection in a two-locus system. Genetics 81:787-802.

- 2008. Linkage disequilibrium - understanding the evolutionary past and mapping the medical future. Nat. Rev. Genetics 9:477-485.

Tepekule, B., H. Uecker, I. Derungs, A. Frenoy, and S. Bonhoeffer. 2017. Modeling antibiotic treatment in hospitals: A systematic approach shows benefits of combination therapy over cycling, mixing, and mono-drug therapies. PLoS Comp. Biol. 13. 
Trindade, S., A. Sousa, K. B. Xavier, F. Dionisio, M. G. Ferreira, and I. Gordo. 2009. Positive epistasis drives the acquisition of multidrug resistance. PLoS Genetics 5.

Turner, P., C. Turner, A. Jankhot, N. Helen, S. J. Lee, N. P. Day, N. J. White, F. Nosten, and D. Goldblatt. 2012. A longitudinal study of Streptococcus pneumoniae carriage in a cohort of infants and their mothers on the Thailand-Myanmar border. PLoS ONE 7.

Weinberger, D. M., K. Trzcinski, Y. Lu, D. Bogaert, A. Brandes, J. Galagan, P. W. Anderson, R. Malley, and M. Lipsitch. 2009. Pneumococcal capsular polysaccharide structure predicts serotype prevalence. PLoS Pathogens 5.

Wilson, D. S. and J. Yoshimura. 1994. On the coexistence of specialists and generalists. Am. Nat. 144:692-707.

Zafar, M. A., S. Hamaguchi, T. Zangari, M. Cammer, and J. N. Weiser. 2017. Capsule type and amount affect shedding and transmission of Streptococcus pneumoniae. mBio 8.

zur Wiesch, P. A., R. Kouyos, S. Abel, W. Viechtbauer, and S. Bonhoeffer. 2014. Cycling empirical antibiotic therapy in hospitals: meta-analysis and models. PLoS Pathogens 10. 


\section{Supplementary Material}

Here we provide more comprehensive details on the different equations, variables, and definitions used in the main text.

\section{Model derivation}

Our focus is on an asymptomatically carried bacteria species in a metapopulation consisting of $N$ populations. Focus upon an arbitrarily chosen population $x$. Let $S^{x}$ and $I_{i j}^{x}$ denote the density of susceptible hosts and $i j$-infections, respectively, at time $t$, where $i$ indicates if the infection is resistant $(i=A)$ or not $(i=a)$ to $\operatorname{drug} A$ and $j$ indicates if the infection is resistant $(j=B)$ or not $(j=b)$ to drug $B$. Susceptible hosts contract $i j$ infections at a per-capita rate $\beta_{i j}^{x} I_{i j}^{x}$, where $\beta_{i j}^{x}$ is a rate constant, while $i j$-infections are naturally cleared at a per-capita rate $\alpha_{i j}^{x}$. Hosts in population $x$ are treated with antibiotics $A, B$, or both in combination, at percapita rates $\tau_{A}^{x}$, $\tau_{B}^{x}$, and $\tau_{A B}^{x}$, respectively. Hosts move from population $x$ to population $y$ at a per-capita rate $m^{x \rightarrow y}$.

The resistance profile of an infection changes through two processes. First, there may be de novo mutation, and so let $\mu_{i}^{x}$ be the per-capita rate at which an infection in population $x$ acquires allele $i$ through mutation. Second, a $i j$-infection may be super-infected by a $k \ell$-strain (Day and Gandon 2012); in this circumstance recombination may occur. Specifically, $k \ell$-strains are transmitted to $i j$-infections at rate $\beta_{k \ell}^{x} I_{k \ell}^{x} I_{i j}^{x}$, whereupon with probability $\sigma$ super-infection occurs. In the event of super-infection, with probability $1-\rho$, recombination does not occur, in which case with equal probability the $i j$-infection either remains unchanged or becomes a $k \ell$-infection. With probability $\rho$, recombination does occur, in which case with equal probability the $i j$-infection becomes either an $i \ell$ - or $k j$-infection. Because our focus is upon the role of population structure, we do not allow for co-infection or within-host competitive differences based upon resistance profiles (e.g., Davies et al. 2019) but these are straightforward extensions. Moreover, at this stage we do not make any further specification of the dynamics of uninfected hosts, be they susceptible or recovered, as doing so is not essential for a qualitative understanding of MDR evolution.

Rather than immediately writing down the set of differential equations corresponding to these epidemiological assumptions, we instead group the terms based upon the four biological processes that are occurring. In particular, the change in $I_{i j}^{X}$ can be written as the sum of:

(1) The net change due to mutation, denoted $\phi \mu_{i j}^{x}$. As an example, focus upon the change in $A b$-infections in population $x$ due to mutation, $\phi \mu_{A b}^{x}$. These infections can increase through mutation in one of two ways: (i) $a b$-infections acquiring allele $A$ at rate $\mu_{A}^{x} I_{a b}^{x}$ or (ii) $A B$-infections acquiring allele $b$ at rate $\mu_{b}^{x} I_{A B}^{x}$. On the other hand, $I_{A b}^{x}$ infections are lost due to mutation whenever they (i) acquire allele $a$ at a per-capita rate $\mu_{a}^{x}$, or (ii) acquire allele $B$ at a per-capita rate $\mu_{B}^{x}$. Combining this information gives the change in $A b$-infections in population $x$ as

$$
\phi \mu_{A b}^{x}=\mu_{A}^{x} I_{a b}^{x}+\mu_{b}^{x} I_{A B}^{x}-\left(\mu_{a}^{x}+\mu_{B}^{x}\right) I_{A b}^{x},
$$

which is mathematically equivalent to

$$
\phi \mu_{A b}^{x}=\mu_{A}^{x}\left(I_{a b}^{x}+I_{A b}^{x}\right)+\mu_{b}^{x}\left(I_{A b}^{x}+I_{A B}^{x}\right)-\mu^{x} I_{A b}^{x},
$$


where $\mu^{x} \equiv \mu_{a}^{x}+\mu_{A}^{x}+\mu_{b}^{x}+\mu_{B}^{x}$ is the per-capita mutation rate in population $x$. The only difference between the two formulations is interpretation: equation (9) shows only mutations which lead to a change in state, whereas equation (10) shows all possible mutations, even those which do not. This is why the per-capita loss term, $\mu^{x}$, in (10) can be considered the total per-capita mutation rate in population $x$. More generally, we can write $\phi \mu_{i j}^{x}$ as

$$
\phi \mu_{i j}^{x} \equiv \mu_{i}^{x}\left(I_{a j}^{x}+I_{A j}^{x}\right)+\mu_{j}^{x}\left(I_{i b}^{x}+I_{i B}^{x}\right)-\mu^{x} I_{i j}^{x}
$$

(2) The net change due to recombination, denoted $\phi \rho_{i j}^{x}$. Specifically, let $\rho_{i}^{x}$ be the per-capita rate at which infections gain allele $i$ through recombination. For example, consider $\rho_{A}^{x}$. In particular, $i j$-infections are challenged by strains carrying allele $A$ at rate $\left(\beta_{A b}^{x} I_{A b}^{x}+\beta_{A B}^{x} I_{A B}^{x}\right) I_{i j}^{x}$. With probability $\sigma$, a superinfection event occurs. Given an superinfection event, with probability $\rho$ recombination happens, in which case with probability $1 / 2$ the recombinant strain $A j$ will replace the $i j$ infection. Thus

$$
\rho_{A}^{x}=\rho \frac{\sigma}{2}\left(\beta_{A b}^{x} I_{A b}^{x}+\beta_{A B}^{x} I_{A B}^{x}\right),
$$

and $i j$-infections acquire allele $A$ in population $x$ at rate $\rho_{A}^{x} I_{i j}^{x}$. Therefore the change in $i j$-infections in population $x$ due to recombination is

$$
\phi \rho_{i j}^{x} \equiv \rho_{i}^{x}\left(I_{a j}^{x}+I_{A j}^{x}\right)+\rho_{j}^{x}\left(I_{i b}^{x}+I_{i B}^{x}\right)-\rho^{x} I_{i j}^{x}
$$

where $\rho^{x}$ is the per-capita rate of recombination in population $x$, that is,

$$
\rho^{x} \equiv \rho \sigma \sum_{k \ell} \beta_{k \ell}^{x} I_{k \ell}^{x}=\rho_{a}^{x}+\rho_{A}^{x}+\rho_{b}^{x}+\rho_{B}^{x}
$$

(3) The net change due to host migration between populations,

$$
-\sum_{y=1}^{N} m^{x \rightarrow y} I_{i j}^{x}+\sum_{y=1}^{N} m^{y \rightarrow x} I_{i j}^{y}
$$

(4) The net change due to per-capita growth,

$$
r_{i j}^{x} \equiv \beta_{i j}^{x} S^{x}-\alpha_{i j}^{x}-\mathbf{1}_{a}(i) \tau_{A}^{x}-\mathbf{1}_{b}(j) \tau_{B}^{x}-\mathbf{1}_{a}(i) \mathbf{1}_{b}(j) \tau_{A B}^{x}-(1-\rho) \frac{\sigma}{2} \sum_{k \ell}\left(\beta_{k \ell}^{x}-\beta_{i j}^{x}\right) I_{k \ell}^{x},
$$

where $\mathbf{1}_{i}(j)$ is an indicator variable and is equal to 1 if $i=j$ and 0 otherwise.

With these four processes in hand, the dynamics of infection densities are given by the system of $4 N$ differential equations

$$
\frac{\mathrm{d} I_{i j}^{x}}{\mathrm{dt}}=\phi \mu_{i j}^{x}+\phi \rho_{i j}^{x}-\sum_{y=1}^{N}\left(m^{x \rightarrow y} I_{i j}^{x}-m^{y \rightarrow x} I_{i j}^{y}\right)+r_{i j}^{x} I_{i j}^{x}, x=1,2, \ldots, N, i \in\{a, A\}, j \in\{b, B\} .
$$




\section{Population LD and MDR}

In what follows, we provide more details for the calculations of population LD and MDR. First, we define the following frequencies of infections in population $x$ as

$$
f_{A}^{x}=\frac{\sum_{j} I_{A j}^{x}}{I^{x}}, \quad f_{B}^{x}=\frac{\sum_{i} I_{i B}^{x}}{I^{x}}, \quad \text { and } \quad f_{i j}^{x}=\frac{I_{i j}^{x}}{I^{x}},
$$

where $I^{x}=\sum_{i j} I_{i j}^{x}$ is the total density of infections in population $x$. Using these definitions, the standard measure of linkage equilibrium in population $x$ is

$$
D^{x}=f_{A B}^{x}-f_{A}^{x} f_{B}^{x},
$$

which is mathematically equivalent to

$$
D^{x}=f_{A B}^{x} f_{a b}^{x}-f_{A b}^{x} f_{a B}^{x} .
$$

The three dynamical equations of interest for studying MDR in population $x$ are

$$
\begin{aligned}
& \frac{\mathrm{d} f_{A}^{x}}{\mathrm{~d} t}=s_{A}^{x} f_{A}^{x}\left(1-f_{A}^{x}\right)+s_{B}^{x} D^{x}+s_{E}^{x} f_{A}^{x}\left(1-f_{A}^{x}\right) \frac{f_{A B}^{x}}{f_{A}^{x}}+\left(\mu_{A}^{x}+\rho_{A}^{x}\right)\left(1-f_{A}^{x}\right)-\left(\mu_{a}^{x}+\rho_{a}^{x}\right) f_{A}^{x} \\
& -\sum_{y=1}^{N} m^{y \rightarrow x} \frac{I^{y}}{I^{x}}\left(f_{A}^{x}-f_{A}^{y}\right) \\
& \frac{\mathrm{d} f_{B}^{x}}{\mathrm{~d} t}=s_{B}^{x} f_{B}^{x}\left(1-f_{B}^{x}\right)+s_{A}^{x} D^{x}+s_{E}^{x} f_{B}^{x}\left(1-f_{B}^{x}\right) \frac{f_{A B}^{x}}{f_{B}^{x}}+\left(\mu_{B}^{x}+\rho_{B}^{x}\right)\left(1-f_{B}^{x}\right)-\left(\mu_{b}^{x}+\rho_{b}^{x}\right) f_{B}^{x} \\
& -\sum_{y=1}^{N} m^{y \rightarrow x} \frac{I^{y}}{I^{x}}\left(f_{B}^{x}-f_{B}^{y}\right) \\
& \frac{\mathrm{d} D^{x}}{\mathrm{dt}}=\left(s_{A}^{x}-s^{x}+s_{B}^{x}-s^{x}\right) D^{x}-\left(\mu^{x}+\rho^{x}\right) D^{x}+s_{E}^{x} f_{A B}^{x} f_{a b}^{x} \\
& -\sum_{y=1}^{N} m^{y \rightarrow x} \frac{I^{y}}{I^{x}}\left(D^{x}-D^{y}-\left(f_{A}^{x}-f_{A}^{y}\right)\left(f_{B}^{x}-f_{B}^{y}\right)\right) .
\end{aligned}
$$

System (19) contains a number of quantities that we now define in more detail. First, the (additive) selection coefficient for resistance to drugs $A$ and $B$ in population $x$ are defined as

$$
s_{A}^{x}=r_{A b}^{x}-r_{a b}^{x} \text { and } s_{B}^{x}=r_{a B}^{x}-r_{a b}^{x},
$$

respectively, while epistasis in population $x$ is $s_{E}^{x}=r_{A B}^{x}+r_{a b}^{x}-r_{A b}^{x}-r_{a B}^{x}$. It follows that we can write each of the per-capita growth rates, $r_{i j}^{x}$, as

$$
r_{i j}^{x}=r^{x}+\mathbf{1}_{A}(i) s_{A}^{x}+\mathbf{1}_{B}(j) s_{B}^{x}+\mathbf{1}_{A}(i) \mathbf{1}_{B}(j) s_{E}^{x} .
$$

This is why $r_{a b}^{x}=r^{x}$ can be thought of as 'baseline' per-capita growth. We define the average selection for resistance in population $x$ as

$$
s^{x}=s_{A}^{x} f_{A}^{x}+s_{B}^{x} f_{B}^{x}+s_{E}^{x} f_{A B}^{x} .
$$

Note that the average per-capita growth rate in population $x$ is therefore $r^{x}+s^{x}$, that is, average per-capita growth rate is the sum of the 'baseline' per-capita growth rate and the average selection for resistance. 


\section{Metapopulation LD and MDR}

Next, consider metapopulation (or total) LD and MDR. First, let $p^{x}=I^{x} / \sum_{j=1}^{N} I^{j}$ be the fraction of total infections in population $x$. Then the metapopulation level equivalents of equations (16) are

$$
\bar{f}_{i}=\sum_{x=1}^{N} p^{x} f_{i}^{x} \quad \text { and } \quad \bar{f}_{i j}=\sum_{x=1}^{N} p^{x} f_{i j}^{x} .
$$

The standard measure of linkage disequilibrium at the level of the metapopulation is

$$
D_{\mathrm{M}}=\bar{f}_{A B}-\bar{f}_{A} \bar{f}_{B} .
$$

which in terms of the population level variables is

$$
D_{\mathrm{M}} \equiv \sum_{x=1}^{N} p^{x} D^{x}+\sum_{x=1}^{N} p^{x} f_{A}^{x} f_{B}^{x}-\left(\sum_{x=1}^{N} p^{x} f_{A}^{x}\right)\left(\sum_{x=1}^{N} p^{x} f_{B}^{x}\right)=\bar{D}+\operatorname{cov}\left(f_{A}, f_{B}\right)
$$

where $\bar{D}$ is the average population $\mathrm{LD}$ and $\operatorname{cov}\left(f_{A}, f_{B}\right)$ is the spatial covariance between frequency of resistance to drug $A$ and frequency of resistance to drug $B$.

Using these variables, the three dynamical equations for studying metapopulation MDR are

$$
\begin{aligned}
\frac{\mathrm{d} \bar{f}_{A}}{\mathrm{~d} t}=\bar{s}_{A} \bar{f}_{A}\left(1-\bar{f}_{A}\right)+\bar{s}_{B} D_{\mathrm{M}}+\bar{s}_{E} \bar{f}_{A}\left(1-\bar{f}_{A}\right) \frac{\bar{f}_{A B}}{\bar{f}_{A}} & \\
& +\left(\bar{\mu}_{A}+\bar{\rho}_{A}\right)\left(1-\bar{f}_{A}\right)-\left(\bar{\mu}_{a}+\bar{\rho}_{a}\right) \bar{f}_{A}+\operatorname{cov}\left(r, f_{A}\right)+\bar{f}_{B} \operatorname{cov}\left(s_{B}, \frac{f_{A B}}{f_{B}}\right), \\
\frac{\mathrm{d} \bar{f}_{B}}{\mathrm{~d} t}= & \bar{s}_{B} \bar{f}_{B}\left(1-\bar{f}_{B}\right)+\bar{s}_{A} D_{\mathrm{M}}+\bar{s}_{E} \bar{f}_{B}\left(1-\bar{f}_{B}\right) \frac{\bar{f}_{A B}}{\bar{f}_{B}} \\
& +\left(\bar{\mu}_{B}+\rho_{B}\right)\left(1-\bar{f}_{B}\right)-\left(\bar{\mu}_{b}+\bar{\rho}_{b}\right) \bar{f}_{B}+\operatorname{cov}\left(r, f_{B}\right)+\bar{f}_{A} \operatorname{cov}\left(s_{A}, \frac{f_{A B}}{f_{A}}\right), \\
\frac{\mathrm{d} D_{\mathrm{M}}}{\mathrm{d} t}=\left(\bar{s}_{A}-\bar{s}+\bar{s}_{B}-\bar{s}\right) D_{\mathrm{M}}-(\bar{\mu}+\bar{\rho}) D_{\mathrm{M}}+\bar{s}_{E} \bar{f}_{a b} \bar{f}_{A B}+\operatorname{cov}(r, D)+\operatorname{coskew}\left(r, f_{A}, f_{B}\right) & \\
& +\sum_{d \in\{A, B\}}\left(1-\bar{f}_{d}\right) \bar{f}_{d} \operatorname{cov}\left(s_{d}, \frac{f_{A B}}{f_{d}}\right)+\left(1-\bar{f}_{A}\right) \Lambda_{A a}-\bar{f}_{A} \Lambda_{a A}+\left(1-\bar{f}_{B}\right) \Lambda_{B b}-\bar{f}_{B} \Lambda_{b B} .
\end{aligned}
$$

Note that in the equation $\mathrm{d} D_{\mathrm{M}} / \mathrm{d} t$, there are terms involving $\Lambda_{i j}$ which we chose to neglect in equation (7) given in the main text. These terms are

$$
\Lambda_{A a}=\operatorname{cov}\left(\mu_{A}+\rho_{A}, \frac{f_{a B}}{1-f_{A}}\right) \quad \text { and } \quad \Lambda_{a A}=\operatorname{cov}\left(\mu_{a}+\rho_{a}, \frac{f_{A B}}{f_{A}}\right)
$$

while

$$
\Lambda_{B b}=\operatorname{cov}\left(\mu_{B}+\rho_{B}, \frac{f_{a B}}{1-f_{B}}\right) \quad \text { and } \quad \Lambda_{b B}=\operatorname{cov}\left(\mu_{b}+\rho_{b}, \frac{f_{A B}}{f_{B}}\right)
$$

Thus the expression

$$
\left(1-\bar{f}_{A}\right) \Lambda_{A a}-\bar{f}_{A} \Lambda_{a A}+\left(1-\bar{f}_{B}\right) \Lambda_{B b}-\bar{f}_{B} \Lambda_{b B}
$$


in the equation $\mathrm{d} D_{\mathrm{M}} / \mathrm{d} t$ is the effect upon $D_{\mathrm{M}}$ of spatial heterogeneity in mutation and recombination rates $\left(\mu_{i}^{x} \neq \mu_{i}^{y}\right.$ and/or $\left.\rho_{i}^{x} \neq \rho_{i}^{y}\right)$ coupled with differences in the proportion of infections with allele $i$ (e.g., $i=A$ or $i=a$ ) that are resistant to the other $\operatorname{drug}(j=B)$. In particular, populations in which infections are more likely to acquire resistance through mutation/recombination disproportionately affect metapopulation LD through an increase in doubly-resistant infections. However, these terms are likely to be quite small because they require that substantial differences in mutation/recombination rates exist between populations. Since these terms are unlikely to be a significant contributor to the dynamics of $D_{\mathrm{M}}$, we ignore them in the main text.

There remains a number of other quantities in system (26) that we now define in more detail. First, the probability that an infection resistant to drug $d$ is found in population $x$ is

$$
p^{x} \frac{f_{d}^{x}}{\bar{f}_{d}}
$$

For example, if we apply our variable definitions, it is straightforward to show that

$$
p^{x} \frac{f_{A}^{x}}{\bar{f}_{A}}=\frac{I_{A b}^{x}+I_{A B}^{x}}{\sum_{y=1}^{N}\left(I_{A b}^{y}+I_{A B}^{y}\right)} .
$$

Next, to compute the metapopulation-level selection coefficients, and mutation/recombination rates, we need to compute the weighted average of the population quantities, where the weights are the probability that an infection of a particular type is in population $x$ (calculated above). Applying this logic, the metapopulation-level selection coefficients and epistasis are

$$
\bar{s}_{i}=\sum_{x=1}^{N} p^{x} \frac{f_{i}^{k}}{\bar{f}_{i}} s_{i}^{x} \quad \text { and } \quad \bar{s}_{E}=\sum_{x=1}^{N} p^{x} \frac{f_{A B}^{x}}{\bar{f}_{A B}} s_{E}^{x} .
$$

The average selection for resistance in the metapopulation is

$$
\bar{s}=\bar{s}_{A} \bar{f}_{A}+\bar{s}_{B} \bar{f}_{B}+\bar{s}_{E} \bar{f}_{A B} .
$$

The per-capita mutation and recombination rates follow similarly. Recall that $\mu_{\ell}$ and $\rho_{\ell}$ are the per-capita rates at which infections gain allele $\ell$. Thus, for example,

$$
\bar{\mu}_{A}=\sum_{x=1}^{N} p^{x} \frac{1-f_{A}^{x}}{1-\bar{f}_{A}} \mu_{A}^{x} \quad \text { and } \quad \bar{\mu}_{a}=\sum_{x=1}^{N} p^{x} \frac{f_{A}^{x}}{\bar{f}_{A}} \mu_{a}^{x} .
$$

Similar calculations can be made to arrive at $\bar{\mu}_{B}, \bar{\mu}_{b}$, and the various $\bar{\rho}_{\ell}$. The total per-capita mutation and recombination rates are

$$
\bar{\mu}=\bar{\mu}_{a}+\bar{\mu}_{A}+\bar{\mu}_{b}+\bar{\mu}_{B} \quad \text { and } \quad \bar{\rho}=\bar{\rho}_{a}+\bar{\rho}_{A}+\bar{\rho}_{b}+\bar{\rho}_{B} .
$$

\subsection{Covariance and coskewness}

Finally, we also use a number of covariance terms and a coskewness terms. Let $\mathbb{E}[c]$ denote the expectation of the quantity $c$. Then applying the definition of covariance, we have

$$
\begin{aligned}
\operatorname{cov}\left(f_{A}, f_{B}\right) & =\mathbb{E}\left[f_{A} f_{B}\right]-\mathbb{E}\left[f_{A}\right] \mathbb{E}\left[f_{B}\right] \\
& =\sum_{x=1}^{N} p^{x} f_{A}^{x} f_{B}^{x}-\left(\sum_{x=1}^{N} p^{x} f_{A}^{x}\right)\left(\sum_{x=1}^{N} p^{x} f_{B}^{x}\right)
\end{aligned}
$$


Following the same procedure, we can calculate $\operatorname{cov}\left(r, f_{A}\right)$ and $\operatorname{cov}\left(r, D_{\mathrm{M}}\right)$. When the covariance involves quantities that also specifically depend upon particular allele(s), the only difference is that when computing the expectation the probability used is the probability that an allele $\ell$ is in population $x$. For example,

$$
\begin{aligned}
\operatorname{cov}\left(s_{A}, \frac{f_{A B}}{f_{A}}\right) & =\mathbb{E}\left[s_{A} \frac{f_{A B}}{f_{A}}\right]-\mathbb{E}\left[s_{A}\right] \mathbb{E}\left[\frac{f_{A B}}{f_{A}}\right] \\
& =\sum_{x=1}^{N} p^{x} \frac{f_{A}^{x}}{\bar{f}_{A}} s_{A}^{x} \frac{f_{A B}^{x}}{f_{A}^{x}}-\left(\sum_{x=1}^{N} p^{x} \frac{f_{A}^{x}}{\bar{f}_{A}} s_{A}^{x}\right)\left(\sum_{x=1}^{N} p^{x} \frac{f_{A}^{x}}{\bar{f}_{A}} \frac{f_{A B}^{x}}{f_{A}^{x}}\right) \\
& =\sum_{x=1}^{N} p^{x} \frac{s_{A}^{x} f_{A B}^{x}}{\bar{f}_{A}}-\left(\sum_{x=1}^{N} p^{x} \frac{f_{A}^{x}}{\bar{f}_{A}} s_{A}^{x}\right)\left(\sum_{x=1}^{N} p^{x} \frac{f_{A B}^{x}}{\bar{f}_{A}}\right) \\
& =\sum_{x=1}^{N} p^{x} \frac{f_{A B}^{x}}{\bar{f}_{A}}\left(s_{A}^{x}-\bar{s}_{A}\right) .
\end{aligned}
$$

The covariance terms involving the recombination and mutation rates follow similarly, with the appropriate exchanges of variables. Finally, we have the coskewness term, which can be calculated as

$$
\begin{aligned}
\operatorname{coskew}\left(r, f_{A}, f_{B}\right) & =\mathbb{E}\left[(r-\mathbb{E}[r])\left(p_{A}-\mathbb{E}\left[f_{A}\right]\right)\left(f_{B}-\mathbb{E}\left[f_{B}\right]\right)\right] \\
& =\operatorname{cov}\left(r, f_{A} f_{B}\right)-\bar{f}_{B} \operatorname{cov}\left(r, f_{A}\right)-\bar{f}_{A} \operatorname{cov}\left(r, f_{B}\right) .
\end{aligned}
$$

\section{Specific examples}

\subsection{Equilibrium analysis of metapopulation consisting of independent pop- ulations}

This is a version of one of the models presented in Lehtinen et al. (2019). The metapopulation consists of $N$ populations. The populations are independent (i.e, there is no migration between populations), and each population is assumed to be of fixed size 1 so $S^{x}=1-\sum_{i j} I_{i j}^{x}$. Resistance is gained and lost through unbiased mutation occurring at rate $\mu$ and there is no recombination. Therefore

$$
\frac{\mathrm{d} I_{i j}^{x}}{\mathrm{~d} t}=\left(\beta_{i j}^{x} S^{x}-\alpha_{i j}^{x}-\mathbf{1}_{a}(i) \tau_{A}^{x}-\mathbf{1}_{b}(j) \tau_{B}^{x}-\left(1-\mathbf{1}_{A}(i) \mathbf{1}_{B}(j)\right) \tau_{A B}^{x}\right) I_{i j}^{x}+\mu\left(\sum_{\ell}\left(I_{\ell j}^{x}+I_{i \ell}^{x}\right)-4 I_{i j}^{x}\right) .
$$

Let $\Delta z_{d}^{x}$ and $\Delta z_{E}^{x}$ denote the contribution of parameter $z$ to the additive selection coefficient (for drug $d$-resistance) and epistasis, respectively, in population $x$. Specifically,

$$
\begin{array}{lll}
\Delta \beta_{A}^{x}=\beta_{A b}^{x}-\beta_{a b}^{x}, & \Delta \beta_{B}^{x}=\beta_{a B}^{x}-\beta_{a b}^{x}, & \Delta \beta_{E}^{x}=\beta_{A B}^{x}+\beta_{a b}^{x}-\beta_{A b}^{x}-\beta_{a B}^{x} \\
\Delta \alpha_{A}^{x}=\alpha_{A b}^{x}-\alpha_{a b}^{x}, & \Delta \alpha_{B}^{x}=\alpha_{a B}^{x}-\alpha_{a b}^{x}, & \Delta \alpha_{E}^{x}=\alpha_{A B}^{x}+\alpha_{a b}^{x}-\alpha_{A b}^{x}-\alpha_{a B}^{x} .
\end{array}
$$

Then if we let $r_{i j}^{x}$ denote the per-capita growth term of an $i j$-infection in subpopulation $x$ (the first term in brackets in equation (36)), we can partition this as

$$
r_{i j}^{x}=r^{x}+\mathbf{1}_{A}(i) s_{A}^{x}+\mathbf{1}_{B}(j) s_{B}^{x}+\mathbf{1}_{A}(i) \mathbf{1}_{B}(j)_{E}^{x}
$$


where

$$
\begin{aligned}
& r^{x}=\beta_{a b}^{x} S^{x}-\alpha_{a b}^{x}-\tau_{A}^{x}-\tau_{B}^{x}-\tau_{A B}^{x} \\
& s_{A}^{x}=\Delta \beta_{A}^{x} S^{x}-\Delta \alpha_{A}^{x}+\tau_{A}^{x} \\
& s_{B}^{x}=\Delta \beta_{B}^{x} S^{x}-\Delta \alpha_{B}^{x}+\tau_{B}^{x} \\
& s_{E}^{x}=\Delta \beta_{E}^{x} S^{x}-\Delta \alpha_{E}^{x}+\tau_{A B}^{x}
\end{aligned}
$$

This notation and formulation differs from that of Lehtinen et al. (2017, 2019) in that they assumed costs were multiplicative, that is,

$$
\beta_{a b}^{x}=\beta^{x}, \quad \beta_{A b}^{x}=\beta^{x} c_{\beta_{A}}^{x}, \quad \beta_{a B}^{x}=\beta^{x} c_{\beta_{B}}^{x}, \quad \beta_{A B}^{x}=\beta^{x} c_{\beta_{A}}^{x} c_{\beta_{B}}^{x}
$$

and

$$
\alpha_{a b}^{x}=\alpha^{x}, \quad \alpha_{A b}^{x}=\frac{\alpha^{x}}{c_{\alpha_{A}}^{x}}, \quad \alpha_{a B}^{x}=\frac{\alpha^{x}}{c_{\alpha_{B}}^{x}}, \quad \alpha_{A B}^{x}=\frac{\alpha^{x}}{c_{\alpha_{A}}^{x} c_{\alpha_{B}}^{x}}
$$

where $0 \leq c_{\beta_{\ell}}^{x} \leq 1$ and $0 \leq c_{\alpha_{\ell}}^{x} \leq 1$. The problem with multiplicative costs is apparent when we compute epistasis,

$$
s_{E}^{x}=\beta^{x}\left(1-c_{\beta_{A}}^{x}\right)\left(1-c_{\beta_{B}}^{x}\right) S^{x}-\alpha^{x} \frac{\left(1-c_{\alpha_{A}}^{x}\right)\left(1-c_{\alpha_{B}}^{x}\right)}{c_{\alpha_{A}}^{x} c_{\alpha_{B}}^{x}}+\tau_{A B}^{x} .
$$

Here we can see that for the model of Lehtinen et al. (2019), only when there are no costs of resistance and no combination treatment will there be no epistasis. Thus transmission costs will produce positive epistasis and duration of carriage costs will produce negative epistasis in the model of Lehtinen et al. (2019).

In Figure 3 we consider three scenarios; whenever possible we choose parameter values to agree with those of Figure 4 in Lehtinen et al. (2019). In each scenario we assume there are 20 independent populations, that the per-capita mutation rate is $\mu=10^{-10}$, and there is no epistasis, $s_{E}^{x}=0$. In subplot $3 \mathbf{a}$, we set $\beta_{a b}^{x}=2$, while duration of carriage varies among populations from $\alpha_{a b}^{x}=0.25$ to $\alpha_{a b}^{x}=1.75$. In subplot $3 \mathbf{b}$ we set $\alpha_{a b}^{x}=0.5$, while transmission varies among populations from $\beta_{a b}^{x}=1$ to $\beta_{a b}^{x}=3$. In both subplots $3 \mathbf{a}$ and $3 \mathbf{b}, \Delta \alpha_{A}^{x}=\Delta \alpha_{B}^{x}=0$, while $\Delta \beta_{A}^{x}=\Delta \beta_{B}^{x}=-0.1$. Finally in subplot $3 \mathrm{c}, \Delta \beta_{A}^{x}=\Delta \beta_{B}^{x}=0$, while duration of carriage varies among populations from $\alpha_{a b}^{x}=0.25$ to $\alpha_{a b}^{x}=1.75$, with $\Delta \alpha_{A}^{x}=\Delta \alpha_{B}^{x}=0.05$.

\subsection{Transient dynamics and MDR in Streptococcus pneumoniae}

Here we use a variant of the model originally proposed by Lehtinen et al. (2017, 2019) in which the populations represent different serotypes. Resistance is gained and lost through unbiased mutation at a per-capita rate $\mu$ and there is no recombination of resistance loci.

Applying these assumptions and using the notation presented with our model from the main text, this yields

$$
\begin{aligned}
\frac{\mathrm{d} I_{i j}^{x}}{\mathrm{~d} t}=\left(\beta_{i j}^{x} v(I, x) S-\alpha_{i j}^{x}-\mathbf{1}_{a}(i) \tau_{A}-\mathbf{1}_{b}(j) \tau_{B}-\left(1-\mathbf{1}_{A}(i) \mathbf{1}_{B}(j)\right) \tau_{A B}\right) & I_{i j}^{x} \\
& +\mu\left(\sum_{\ell}\left(I_{\ell j}^{x}+I_{i \ell}^{x}\right)-4 I_{i j}^{x}\right)
\end{aligned}
$$


where

$$
v(I, x)=\left(1-\left[\frac{\sum_{i j} I_{i j}^{x}}{\sum_{k=1}^{N} \sum_{i j} I_{i j}^{k}}-\frac{1}{N}\right]\right)^{\omega}
$$

is a balancing function intended to mimic the stabilizing effect adaptive host immunity has upon serotype diversity ( $\omega$ controls the strength of this effect; see Lehtinen et al. (2017)). Note that the treatment rates are assumed to be independent of serotype.

If we let $r_{i j}^{x}$ denote the per-capita growth term of an $i j$-infection belonging to serotype $x$ (the first term in brackets in equation (43), we can partition this as

$$
r_{i j}^{x}=r^{x}+\mathbf{1}_{A}(i) s_{A}^{x}+\mathbf{1}_{B}(j) s_{B}^{x}+\mathbf{1}_{A}(i) \mathbf{1}_{B}(j) s_{E}^{x}
$$

where if we use the notation introduced in equation (37),

$$
\begin{aligned}
r^{x} & =\beta_{a b}^{x} v(I, x) S-\alpha_{a b}^{x}-\tau_{A}-\tau_{B}-\tau_{A B} \\
s_{A}^{x} & =\Delta \beta_{A}^{x} v(I, x) S-\Delta \alpha_{A}^{x}+\tau_{A} \\
s_{B}^{x} & =\Delta \beta_{B}^{x} v(I, x) S-\Delta \alpha_{B}^{x}+\tau_{B} \\
s_{E}^{x} & =\Delta \beta_{E}^{x} v(I, x) S-\Delta \alpha_{E}^{x}+\tau_{A B}
\end{aligned}
$$

For simplicity we keep total population size constant, and so set $S=1-\sum_{x=1}^{N} \sum_{i j} I_{i j}^{x}$.

The simulations in Figure 5 assume the metapopulation is initially treated at per-capita rates $\left(\tau_{A}, \tau_{B}, \tau_{A B}\right)=(0.12,0,0)$, until $t=1000$ when these rates switch to $\left(\tau_{A}, \tau_{B}, \tau_{A B}\right)=(0.07,0.1,0)$. Other parameters values used are $N=12, \omega=3, \Delta \beta_{A}^{x}=\Delta \beta_{B}^{x}=-0.2, \Delta \alpha_{A}^{x}=\Delta \alpha_{B}^{x}=0.05$ and $\mu=10^{-8}$. Finally, because Streptococcus serotypes differ based upon duration of carriage and transmissibility, and there is evidence of a positive correlation between the two (Weinberger et al. 2009; Zafar et al.2017), $\alpha_{a b}^{x}$ was chosen to assume evenly spaced parameter values from $\alpha_{a b}^{x}=0.2$ to $\alpha_{a b}^{x}=0.7$, while $\beta_{a b}^{x}$ was chosen to assume evenly spaced parameter values from $\beta_{a b}^{x}=3.25$ to $\beta_{a b}^{x}=3$. Cost epistasis, when it is present, is assumed to solely effect transmissibility (i.e., $\Delta \alpha_{E}^{x}=0$ ). When there is positive epistasis, $\Delta \beta_{E}^{x}=0.065$, whereas for negative epistasis, $\Delta \beta_{E}^{x}=-0.015$.

\subsection{Contrasting drug prescription strategies in a hospital-community set- ting}

When we model the hospital and community, we use equation (2) and assume the susceptible host density is controlled by

$$
\begin{aligned}
\frac{\mathrm{d} S^{x}}{\mathrm{~d} t}=\theta^{x}-\kappa S^{x}-m^{x \rightarrow} & S^{x}+m^{y \rightarrow x} S^{y}-\sum_{i j} \beta_{i j}^{x} I_{i j}^{x} S^{x} \\
& +\sum_{i j}\left(\alpha_{i j}^{x}-\kappa\right) I_{i j}^{x}+\sum_{i j}\left(\tau_{A}^{x} \mathbf{1}_{a}(i)+\tau_{B}^{x} \mathbf{1}_{b}(j)+\tau_{A B}^{x}\left(1-\mathbf{1}_{A}(i) \mathbf{1}_{B}(j)\right)\right) I_{i j}^{x}
\end{aligned}
$$

where $\theta^{x}$ is the influx of new hosts and $\kappa$ is the background mortality rate.

In the hospital/community model, we assume population $C$ is the 'community' and population $H$ is the 'hospital'. Therefore we let $\theta^{H}=0$, and $m^{C \rightarrow H}=m / \sum_{i j} I_{i j}^{C}$ be the rate at which individuals are admitted to the hospital, which is independent of population size. Individuals exit 
the hospital at a constant rate $m^{H \rightarrow C}$, so they spend on average $1 / m^{H \rightarrow C}$ time units in hospital (assuming background mortality is low). The specification of the migration rates in this way allows us to ensure the 'community' is always much larger than the 'hospital'. In Figure 6, we assumed that the total prescription rate per population, $\tau^{C}$ or $\tau^{H}$, was the same for each strategy, and that drugs are prescribed at $15 \times$ the rate in the hospital versus the community, that is, $\tau^{H}=15 \tau^{C}$. For 'mixing', this means $\left(\tau_{A}^{x}, \tau_{B}^{x}, \tau_{A B}^{x}\right)=\left(\tau^{x} / 2, \tau^{x} / 2,0\right)$, whereas for 'combination', this means $\left(\tau_{A}^{x}, \tau_{B}^{x}, \tau_{A B}^{x}\right)=\left(0,0, \tau^{x}\right)$. Finally, for 'cycling' drug $A$ and $B$ were rotated from hospital to community every 50 time units so that either $\left(\tau_{A}^{x}, \tau_{B}^{x}, \tau_{A B}^{x}\right)=\left(\tau^{x}, 0,0\right)$ or $\left(\tau_{A}^{x}, \tau_{B}^{x}, \tau_{A B}^{x}\right)=\left(0, \tau^{x}, 0\right)$ depending on the rotation. Therefore in Figure 6 the period is of length $T=100$. In Figure 6 , we numerically integrate the system until $t=35,000$; the final state at $t=35,000$ is then what is plotted for the combination and mixing scenarios, whereas for cycling we plot the average state across the last two rotations (i.e., the average state over the final period, from $t=34,900$ to $t=35,000$ ). Figure $\mathrm{S} 1$ shows how changing the length of time between drug rotations affects the evolution of single- and multi-drug resistance. Specifically, when drug rotations are frequent (with period of $T=1$ ), cycling behaves like mixing and so positive LD is produced (Fig. S1 a). As drug rotations become less frequent (period of $T=24$ and $T=160$ ), cycling generates a negative covariance in selection, which in turn produces negative LD (Fig. S1a). Thus when drug rotations are more frequent, single-drug resistance is delayed and emerges at higher treatment rates, but the evolution of MDR occurs at lower treatment rates (Fig. S1b). When drug rotations are infrequent, single-drug resistance emerges at lower treatment rates, but MDR evolution is delayed, emerging at higher treatment rates (Fig. S1b). Parameters used in Figure 6 and Figure S1 were $\beta_{a b}^{x}=2, \Delta \beta_{A}^{x}=\Delta \beta_{B}^{x}=-0.4, \alpha_{a b}^{x}=0.1, \Delta \alpha_{A}^{x}=\Delta \alpha_{B}^{x}=0.02, \kappa=0.01, \theta^{C}=0.2, \theta^{H}=0$, $m^{H \rightarrow C}=0.5, m=0.2, \mu=10^{-7}, \sigma=0$. 

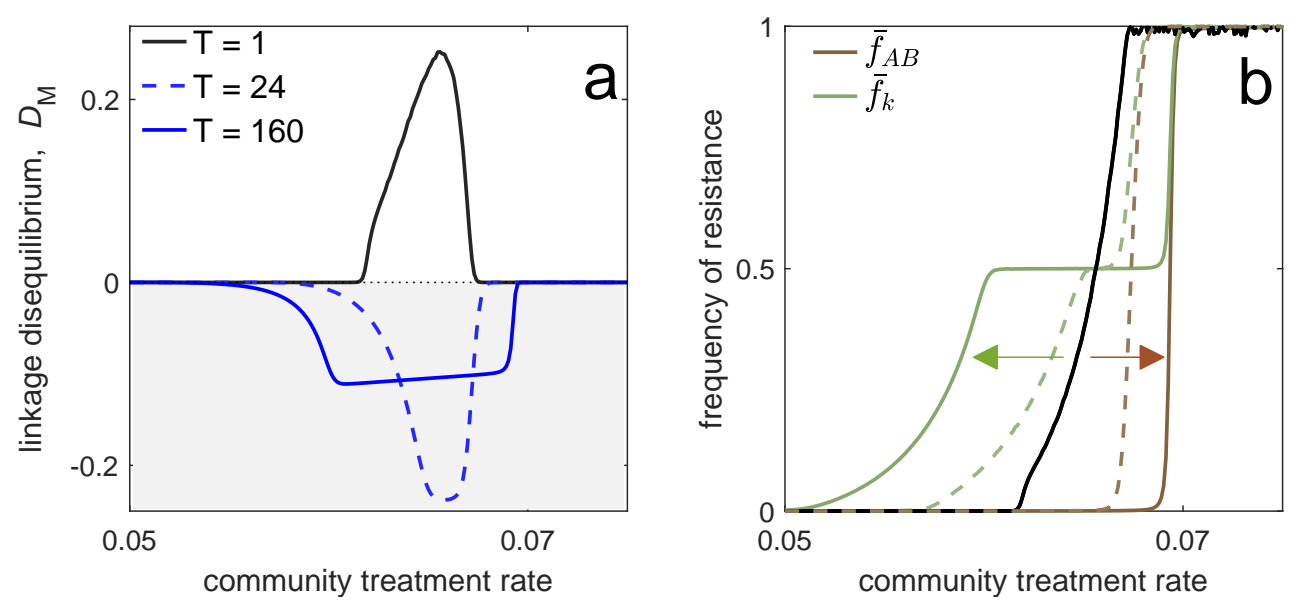

Figure S1: Time between drug rotations affects the evolution of both single- and multi-drug resistance. When drugs are rotated every 0.5 time units (period of $T=1$; black curves), cycling behaves like mixing and positive LD is generated. As we increase the time between rotations (period of $T=24$ and $T=160$ ), a negative covariance in selection is generated, producing negative LD (dashed and solid blue curves). In panel a we show the metapopulation $\mathrm{LD}, D_{\mathrm{M}}$, while in panel b, we show the frequency of resistance in the metapopulation. When drugs are rotated frequently, single drug resistance emerges at higher treatment rates but MDR emerges at lower treatment rates, as compared to when drugs are rotated infrequently. Thus there is a trade-off (indicated by arrows in panel $\mathbf{b}$ ) associated with time between drug rotations: we can delay single drug resistance but promote MDR (frequent drug rotations), or delay MDR but promote single drug resistance (infrequent drug rotations). In all cases, we integrated the system until $t=10^{4}$, then averaged the system state over the final two rotations (i.e., over a single period). The remaining parameter values are provided in Sup. Mat. 4.3. 


\begin{tabular}{|c|c|}
\hline Symbol & Description \\
\hline$I_{i j}^{x}$ & $\begin{array}{l}\text { Density of } i j \text {-infections in population } x \text {, where } i=A \text { (resp. } i=a \text { ) if } \\
\text { infection is resistant (resp. sensitive) to } \operatorname{drug} A \text { and } j=B \text { (resp. } j=b \text { ) } \\
\text { if infection is resistant (resp. sensitive) to } \operatorname{drug} B \text {. }\end{array}$ \\
\hline$I^{x}$ & Density of total infections in population $x$. \\
\hline$f_{d}^{x}, \bar{f}_{d}$ & $\begin{array}{l}\text { Frequency of infections resistant to drug } d \text { in population } x \text { and the } \\
\text { metapopulation, respectively. }\end{array}$ \\
\hline$D^{x}, \bar{D}, D_{\mathrm{M}}$ & $\begin{array}{l}\text { Linkage disequilibrium (LD) in population } x \text {, average LD across pop- } \\
\text { ulations and metapopulation LD, respectively. }\end{array}$ \\
\hline$m^{x \rightarrow y}$ & Per-capita rate at which hosts migrate from population $x$ to $y$. \\
\hline$r^{x}, \bar{r}$ & $\begin{array}{l}\text { Per-capita growth rate of sensitive infections in population } x \text { (or } \\
\text { 'baseline' per-capita growth rate) and average across populations, re- } \\
\text { spectively. }\end{array}$ \\
\hline$s_{d}^{x}, \bar{s}_{d}$ & $\begin{array}{l}\text { Additive selection coefficient for resistance to drug } d \text { in population } x \\
\text { and average selection across populations, respectively. }\end{array}$ \\
\hline$s_{E}^{x}, \bar{s}_{E}$ & $\begin{array}{l}\text { Fitness epistasis across drug resistance loci in population } x \text { and aver- } \\
\text { age across populations, respectively. }\end{array}$ \\
\hline$\phi \mu_{i j}^{x}, \phi \rho_{i j}^{x}$ & $\begin{array}{l}\text { Net change in } i j \text {-infections in population } x \text { due to mutation or re- } \\
\text { combination, respectively. }\end{array}$ \\
\hline$\mu_{i}^{x}, \bar{\mu}_{i}$ & $\begin{array}{l}\text { Per-capita rate at which mutations generate allele } i \text { in population } x \\
\text { and average across populations, respectively. }\end{array}$ \\
\hline$\rho_{i}^{x}, \bar{\rho}_{i}$ & $\begin{array}{l}\text { Per-capita rate at which recombination leads to gain of allele } i \text { in pop- } \\
\text { ulation } x \text { and average across populations, respectively. }\end{array}$ \\
\hline$s^{x}, \bar{s}$ & $\begin{array}{l}\text { Average selection for drug resistance in population } x \text { and average } \\
\text { across populations, respectively.. }\end{array}$ \\
\hline $\operatorname{cov}(X, Y)$ & $\begin{array}{l}\text { Covariance between the variables } X \text { and } Y \text {, that is, } \operatorname{cov}(X, Y)= \\
\mathbb{E}[X Y]-\mathbb{E}[X] \mathbb{E}[Y] \text {, where } \mathbb{E}[X] \text { denotes the expectation of quantity } X \text {. }\end{array}$ \\
\hline $\operatorname{coskew}(X, Y, Z)$ & $\begin{array}{l}\text { Coskewness between the quantities } X, \quad Y, \quad Z \text {, that is, } \\
\operatorname{coskew}(X, Y, Z)=\mathbb{E}[(X-\mathbb{E}[X])(Y-\mathbb{E}[Y])(Z-\mathbb{E}[Z])] .\end{array}$ \\
\hline
\end{tabular}

Table 1: Notation used in main text. In all cases, a quantity indexed with a superscript $x$ is the population $x$ quantity, whereas the absence of a superscript $x$ implies the quantity is for the metapopulation. 